\title{
Eficiencia isoterma de los modelos de ventilación exterior en patios de edificios residenciales. Estudio de casos
}

\section{Isothermal efficiency for outdoor ventilation models of courtyards in residential buildings. Case study}

\author{
M. A. Padilla-Marcos $^{(*)}$, J. Feijó-Muñoz ${ }^{(*)}$, A. Meiss ${ }^{(*)}$
}

\section{RESUMEN}

Muchos edificios han sido diseñados y construidos con patios interiores para satisfacer exigencias de habitabilidad, ya que estos espacios surten de luz y aire a los locales interiores. Sin embargo, la calidad del aire confinado en muchos de ellos es insalubre para el uso humano debido a la mermada capacidad que tienen para renovar su aire. El propósito del estudio es catalogar diferentes modelos de patio interior en edificios aislados, en condiciones isotermas, según sus dimensiones. El objetivo consiste en cuantificar la eficiencia en la renovación del aire en cada modelo y analizar la influencia de sus dimensiones en unas condiciones externas controladas. Adicionalmente, se comparan las normativas vigentes en España con los resultados así obtenidos. Como conclusión se determina la razón de cómo dicha eficiencia de la ventilación de patios interiores de los edificios desciende en función de su diseño.

Palabras clave: Patio interior; eficiencia de la renovación exterior; edad del aire exterior; calidad del aire exterior.

\section{ABSTRACT}

Many buildings have been designed and built with courtyards to supply the light and healthy air demands to the dwellings. However, confined air quality in many of them is unhealthy for human use due to the diminished ability to renew the air. The purpose of the study is to catalog different models of isolated building courtyards under isothermal conditions, according to their dimensions. The goal is to quantify the efficiency of air exchange in each model and analyze the influence of size in controlled external conditions. In addition, current regulations in Spain are compared with the obtained results. In conclusion, the efficiency of ventilation ratio for courtyards in buildings depends on its design.

Keywords: Courtyard; efficiency of the outdoor ventilation; age of the outdoor air; quality of the outdoor air.

(*) GIR-Arquitectura \& Energía. ETSA-Universidad de Valladolid (España).

Persona de contacto/Corresponding author: miguelangel.padilla@uva.es (M. A. Padilla-Marcos)

Cómo citar este artículo/Citation: Padilla-Marcos, M. A., Feijó-Muñoz, J., Meiss, A. (2015). Eficiencia isoterma de los modelos de ventilación exterior en patios de edificios residenciales. Estudio de casos. Informes de la Construcción, 67(540): e121, doi: http://dx.doi. org/10.3989/ic.15.029.

Licencia/License: Salvo indicación contraria, todos los contenidos de la edición electrónica de Informes de la Construcción se distribuyen bajo una licencia de uso y distribución Creative Commons Reconocimiento no Comercial 3.o. España (cc-by-nc). 


\section{INTRODUCCIÓN}

Las urbes se definen por la configuración de la malla urbana, con edificios y vacíos edificatorios (calles, corredores, zonas verdes y patios interiores, abiertos o de manzana, entre otros), denominados «espacios exteriores». El acceso de aire a estos espacios se ve influenciado por sus condiciones geométricas y dimensionales respecto a los edificios de su entorno (1). La importancia de la renovación del aire en los espacios exteriores, por influencia del aire libre, radica en la conservación de su calidad, dado que surten de aire «limpio» a los habitáculos interiores (2) (3).

Los diseños arquitectónicos elegidos por los proyectistas atienden a criterios estéticos, funcionales u ocupacionales. La necesidad de reducir el coste de las edificaciones aprovechando la superficie edificable del suelo urbano implica la reducción de los espacios exteriores. Lo que conlleva la dificultad de renovación de su aire al reducirse la superficie de intercambio con el aire libre y la exposición a la acción del viento (4). La calidad del aire en los espacios exteriores es inferior a la del aire «limpio» procedente de las áreas suburbanas, pues depende de la capacidad de renovación del perfil urbano.

Esta capacidad de ventilación de los espacios entre construcciones está estrechamente relacionada con la disposición de los edificios frente a la acción dinámica del viento y las fuerzas y tensiones de arrastre por acción de la viscosidad (que promueven la turbulencia y facilitan el acceso del aire a las zonas protegidas) (5).

El concepto de eficiencia de la renovación (6) mediante el concepto de la edad del aire permite identificar la capacidad de ventilación de un espacio para mantener unas óptimas condiciones de calidad (7) (8). Estos indicadores han sido ampliamente desarrollados para su aplicación en espacios interiores, siendo transferible su aplicación sobre modelos de espacio exterior.

El objetivo del presente trabajo consiste en analizar comparativamente la idoneidad de diferentes dimensiones de patio interior (Figura 1) ante la renovación del aire contenido bajo unas condiciones de contorno idénticas. En este proceso, la eficiencia de la ventilación depende de un elevado número de variables físicas del aire y geométricas derivadas de la configuración edificatoria del entorno.

Este estudio analiza independientemente diferentes edificios aislados con patio interior simulados en un entorno llano y unas mínimas condiciones de rugosidad (9). De este modo se obtiene la influencia de los parámetros dimensionales de este tipo de espacios exteriores en relación a la calidad del aire (10).

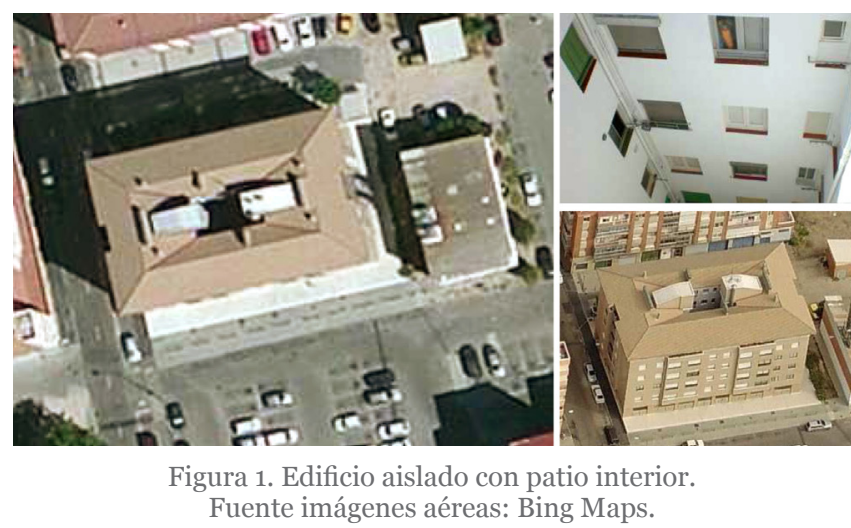

\section{PATIOS INTERIORES EN ESPAÑA}

El diseño de la ventilación de los espacios interiores de los edificios en España se define según la normativa estatal para el conjunto de las Comunidades Autónomas mediante el Código Técnico de la Edificación (CTE) (11). Asimismo, las normas técnicas locales que se centran en los criterios de uso y soleamiento de los recintos interiores que abren a los patios interiores (también llamados «de luces»), en función de su ámbito geográfico.

Por esta razón, el propósito del estudio radica en la verificación de las condiciones de ventilación de los patios interiores definidos por las diferentes normativas vigentes según el concepto de la eficiencia de la ventilación.

Las principales normas de referencia a nivel nacional y local (en importantes capitales de provincia, como Madrid, Barcelona, Sevilla, Bilbao y Valladolid) de las condiciones de diseño, en cuanto a ventilación, impuestas a los patios interiores son (Tabla 1):

- El CTE (RD 314/2006) (11) en el artículo 3.2.1.1 del apartado 3 (Calidad del aire interior) del Documento Básico HS Salubridad prescribe que: «En ausencia de norma urbanística que regule sus dimensiones, los espacios exteriores y los patios [...] deben permitir que en su planta se pueda inscribir un círculo cuyo diámetro sea igual a un tercio de la altura del cerramiento más bajo de los que lo delimitan y no menor que $3 \mathrm{~m}$ ».

- La normativa técnica para el diseño de las Viviendas de Protección Oficial (VPO) (12) es considerada como norma de referencia en España para el diseño de dichos edificios de viviendas. En su ordenanza $13^{\mathrm{a}}$, «Dimensiones de los patios», se establecen las dimensiones mínimas de los patios según el uso de los espacios que ventilan a ellos.

- El Plan General de Ordenación Urbana (PGOU) (13) para la ciudad de Madrid de 1997 (PG-M) define las características, composición y dimensiones de los patios de los edificios en la sección tercera, condiciones higiénicas de los edificios del capítulo 6.7 del Título 1. En su artículo 6.7.12, «Patios» se definen las dimensiones mínimas del patio atendiendo al uso de los locales que abren a él.

- El Decreto 141/2012 sobre las «Condiciones mínimas de habitabilidad de las viviendas y la cédula de habitabilidad» de Cataluña (14) (VP-C) define las condiciones de diseño de los patios para la ciudad de Barcelona según el uso de los locales que abren al patio. El PGOU de Barcelona (15) (PG-C) no discrimina entre usos y aporta unas condiciones mínimas a cumplir.

- La Orden 21 de julio de 2008, «sobre normativa técnica de diseño y calidad aplicable a las viviendas protegidas en la Comunidad Autónoma de Andalucía...» (16) (VP-A) establece un diámetro mínimo de 3 metros y una superficie mínima de $12 \mathrm{~m}^{2}$. Sin embargo, el PGOU de Sevilla (17) (PG-S) establece distintas dimensiones según el tipo de uso de los locales que abren al patio incluyendo el uso tradicional del patio como habitable.

- En el apartado 4.2.b del anexo I de la normativa para el diseño de las VPO para el País Vasco (18) (VP-V), se definen las dimensiones mínimas de los patios según uso de las 
Tabla 1. Principales normativas para el diseño de patios en España.

\begin{tabular}{|c|c|c|c|c|}
\hline Norma & Uso interior & \begin{tabular}{|c|}
$\begin{array}{c}\text { Diámetro círculo } \\
\text { mínimo }\left(\varnothing_{\min }\right)\end{array}$ \\
\end{tabular} & $\begin{array}{c}\text { Superficie } \\
\text { mínima }\left(S_{\min }\right)\end{array}$ & Condiciones \\
\hline CTE & Todos & $\begin{array}{l}\mathrm{H} / 3 \\
3 \mathrm{~m}\end{array}$ & - & \\
\hline \multirow{3}{*}{ VPO } & Sólo dormitorios & $\begin{array}{c}\mathrm{O}, 3 \cdot \mathrm{H} \\
3 \mathrm{~m}\end{array}$ & $\begin{array}{l}\mathrm{H}^{2} / 8 \\
12 \mathrm{~m}^{2}\end{array}$ & \\
\hline & Cocinas sin dormitorios & $\begin{array}{c}\mathrm{O}, 2 \cdot \mathrm{H} \\
3 \mathrm{~m}\end{array}$ & $\begin{array}{l}\mathrm{H}^{2} / 10 \\
12 \mathrm{~m}^{2}\end{array}$ & \\
\hline & $\begin{array}{l}\text { Sin cocinas ni } \\
\text { dormitorios }\end{array}$ & $\begin{array}{l}\mathrm{O}, 15 \cdot \mathrm{H} \\
3 \mathrm{~m}\end{array}$ & $\begin{array}{c}\mathrm{H}^{2} / 2 \mathrm{O} \\
9 \mathrm{~m}^{2}\end{array}$ & \\
\hline \multirow{3}{*}{ PGOU- Madrid } & Habitables sin cocinas & $\begin{array}{l}\mathrm{H} / 3 \\
3 \mathrm{~m}\end{array}$ & - & \\
\hline & Cocinas & $\begin{array}{l}\mathrm{H} / 4 \\
3 \mathrm{~m}\end{array}$ & - & \\
\hline & No habitables & $\begin{array}{l}\mathrm{H} / 5 \\
3 \mathrm{~m}\end{array}$ & - & \\
\hline \multirow{3}{*}{ VPO- Cataluña } & Dormitorios & $3 \mathrm{~m}$ & $9 \mathrm{~m}^{2}$ & $\begin{aligned} S_{\text {min }}= & 9+1,8 \cdot(H / 3-3) \\
& \text { si } \mathrm{H}>9 \mathrm{~m}\end{aligned}$ \\
\hline & $\begin{array}{l}\text { Cocinas, baños y } \\
\text { escaleras }\end{array}$ & $2,5 \mathrm{~m}$ & $6 \mathrm{~m}^{2}$ & $\begin{array}{c}S_{\text {min }}=6+0,9 \cdot(H / 3-3) \\
\text { si H }>9 \mathrm{~m}\end{array}$ \\
\hline & $\begin{array}{l}\text { Cajas de escaleras y } \\
\text { cámaras higiénicas }\end{array}$ & $1,8 \mathrm{~m}$ & $0,9 \mathrm{~m}^{2}$ & $\begin{array}{c}\varnothing_{\min }=1,8+0,1 \cdot(H / 3-3 \\
\text { si H }>9 \mathrm{~m}\end{array}$ \\
\hline \multirow{3}{*}{$\begin{array}{l}\text { PGOU- Barce- } \\
\text { lona }\end{array}$} & \multirow{3}{*}{ Todos } & \multirow{3}{*}{$\begin{array}{l}\mathrm{H} / 7 \\
2 \mathrm{~m}\end{array}$} & $5 \mathrm{~m}^{2}$ & si $\mathrm{H} \leq 6 \mathrm{~m}$. \\
\hline & & & $2 \cdot H / 3+1$ & si $6 \mathrm{~m}<\mathrm{H} \leq 21 \mathrm{~m}$ \\
\hline & & & $17 \mathrm{~m}^{2}$ & $\mathrm{Si} \mathrm{H}>21 \mathrm{~m}$ \\
\hline VPO-Andalucía & Todos & $3 \mathrm{~m}$ & $12 \mathrm{~m}^{2}$ & \\
\hline \multirow{3}{*}{ PGOU- Sevilla } & Sin cocinas & $\begin{array}{c}\mathrm{H} / 3 \\
3,3 \mathrm{~m}\end{array}$ & - & \\
\hline & Sólo cocinas & $\begin{array}{l}\mathrm{H} / 4 \\
3 \mathrm{~m}\end{array}$ & - & \\
\hline & Patios de ventilación & $\begin{array}{l}\mathrm{H} / 5 \\
2 \mathrm{~m}\end{array}$ & - & \\
\hline \multirow{4}{*}{$\begin{array}{l}\text { VPO- } \\
\text { País Vasco }\end{array}$} & Sólo estar y/o comedor & $3 \mathrm{~m}$ & - & \\
\hline & Dormitorios y cocinas & $\begin{array}{c}\mathrm{H} \\
3 \mathrm{~m} \\
\end{array}$ & - & \\
\hline & Baños & $\begin{array}{c}3 \cdot \mathrm{H} / 2 \mathrm{O} \\
3 \mathrm{~m}\end{array}$ & - & \\
\hline & Escaleras & $\begin{array}{c}3 \cdot \mathrm{H} / 2 \mathrm{O} \\
2 \mathrm{~m}\end{array}$ & - & \\
\hline \multirow{4}{*}{ PGOU- Bilbao } & Todos & $5 \mathrm{~m}$ & $25 \mathrm{~m}^{2}$ & $\begin{array}{c}\emptyset_{\text {min }}=(H / 2-9)+5 \\
\text { si } H>18 \mathrm{~m}\end{array}$ \\
\hline & \multirow{3}{*}{ Baños y escaleras } & $3 \mathrm{~m}$ & $9 \mathrm{~m}^{2}$ & si $\mathrm{H}<20 \mathrm{~m}$ \\
\hline & & $4 \mathrm{~m}$ & $16 \mathrm{~m}^{2}$ & si $20 \mathrm{~m}<\mathrm{H}<24 \mathrm{~m}$ \\
\hline & & $5 \mathrm{~m}$ & $25 \mathrm{~m}^{2}$ & si $\mathrm{H}>24 \mathrm{~m}$ \\
\hline
\end{tabular}

estancias adosadas. El PGOU de Bilbao (19) (PG-Bi), en su artículo 7.3.1.3. del título séptimo, capítulo tercero dentro del tomo II se indican las dimensiones mínimas que han de cumplir los patios de parcela o «de luces».

- El PGOU de Valladolid (20) establece las condiciones de diseño de los patios según lo dispuesto en la normativa nacional aplicable a las VPO.

\section{EFICIENCIA DE LA VENTILACIÓN EXTERIOR}

La forma del volumen de aire de un espacio acotado condiciona el desplazamiento del aire en su interior. El camino que sigue el aire en su proceso de renovación depende de la posición de las aberturas de entrada de aire «limpio» y salida del aire «viciado» así como de los obstáculos (). La trayectoria se analiza mediante el concepto de la edad del aire (6) (21). Este parámetro posibilita el cálculo de la eficiencia de la ven- tilación de un espacio con un caudal de renovación continuo, lo que permite analizar la idoneidad de la forma del espacio frente al proceso de renovación por mezcla con el aire nuevo suministrado (22).

Para el análisis de la eficiencia en los espacios exteriores, donde las superficies de admisión y extracción no se encuentran delimitadas físicamente, se requiere la definición de un dominio de control. El dominio de control acota virtualmente un volumen de aire mayor con el fin de identificar los límites por donde el aire entra y sale de un modelo abierto (23).

Para el presente estudio se han analizado los modelos de patio en dos escalas (Figura 2):

- el dominio de control envuelve todo el edificio para obtener el valor de la eficiencia de la ventilación en el patio dependiente del comportamiento del aire en su entorno y; 


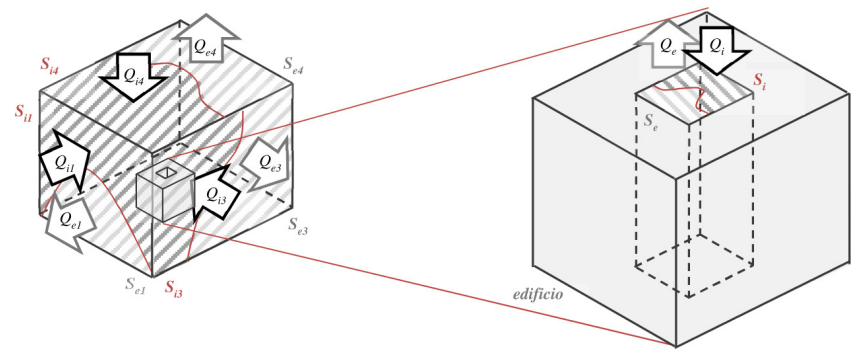

(a) dominio de control envolvente del edificio

(b) dominio de control del patio

Figura 2. Dominio de control para el cálculo de la eficiencia.

- el dominio de control delimita el patio, lo que aporta la información de su eficiencia analizando el comportamiento del aire en su interior por influencia de la velocidad y turbulencia en su embocadura.

En ambos tipos de dominio de control se determinan y delimitan las superficies por donde el aire accede y sale libremente, evaluando los caudales de ventilación bajo un modelo numérico RANS (Reynolds Average Navier-Stokes) (24) con valores promedio de la velocidad del viento en entornos urbanos.

El concepto de la eficiencia de la ventilación en los espacios exteriores [1] (6) se basa en la relación existente entre el tiempo mínimo [2] que tarda el volumen de aire del espacio en renovarse a razón del caudal de ventilación según la ecuación de continuidad y la edad media del aire en el espacio [3] (21).

$$
\begin{gathered}
\varepsilon^{a}=\frac{\tau_{e}}{\tau_{t}} \leq 1 \\
\tau_{e}=\frac{V}{Q_{e q}} \\
\tau_{t}=2 \cdot\langle\bar{\tau}\rangle
\end{gathered}
$$

Siendo: $\tau_{e}$ el tiempo mínimo de intercambio (s); $V$ el volumen de aire analizado $\left(\mathrm{m}^{3}\right) ; Q_{e q}$ el flujo equilibrado de aire en el dominio de control $\left(\mathrm{m}^{3} / \mathrm{s}\right) ; \tau_{t}$ la edad media del aire en la salida (tiempo de residencia del aire en el dominio de control) (s); la edad media del volumen de aire (s) y; $\varepsilon^{a}$ la eficiencia de renovación del aire exterior en tanto por uno.

El caudal equilibrado es el caudal medio de admisión y extracción deducidas las pérdidas de flujo en los modelos reales y a escala o la asunción de la desviación marginal en la convergencia de los cálculos simulados. La aplicación de la ecuación de continuidad permite el equilibrado entre los flujos de admisión y salida del modelo simulado. El dominio de control que envuelve el entorno inmediato del edificio está influenciado por el caudal correspondiente a la proyección de su límite frontal sobre el perfil de velocidades logarítmico urbano [4].

$$
Q_{e q}=w \cdot h^{2} \cdot \frac{U^{*}}{\mathrm{~K}} \cdot\left(\ln \left(\frac{h}{Z_{\mathrm{o}}}\right)-1\right)
$$

Dónde: $w$ es el ancho del dominio de control (m); $h$ es la altura del dominio de control $(\mathrm{m})$; $U^{*}$ la velocidad de fricción $(\mathrm{m} / \mathrm{s}) ; \mathrm{K}$ constante empírica de Von $\operatorname{Karman}(\mathrm{K} \approx 0,41)$ y; $z_{\mathrm{o}}$ la altura de rugosidad del medio físico (m).

\section{VALIDACIÓN DE LA CONFIGURACIÓN CFD}

La validación CFD (25) se realiza simulando numéricamente la sección interior de un túnel de viento BLASIUS donde se produce la afección del edificio sobre el flujo de aire, simplificando las propiedades dinámicas y turbulentas del flujo mediante la definición de perfiles. Las ecuaciones que definen los perfiles de viento han sido validadas experimentalmente en el Environmental Wind Tunnel Laboratory (EWTL) del Instituto de Meteorología de la Universidad de Hamburgo mediante los dataset disponibles del proyecto CEDVAL (http://www. mi.zmaw.de/index.php?id=432). Su fin es la corroboración de las hipótesis tomadas en la simplificación del procedimiento de cálculo (26) y que sirven de soporte al CFD.

La validación se realiza en dos fases dada la complejidad de simulación de un medio urbano abierto a las condiciones de contorno atmosféricas. La primera fase consiste en identificar las variables que intervienen en la dinámica de fluidos para un edificio sencillo aislado y su simplificación para definir unos perfiles que simulen tales condiciones a escala real (27) (28). La segunda fase recrea la configuración sobre geometrías urbanas compuestas por edificios con patio.

Para la primera fase de validación se emplea un edificio cúbico centrado en la sección de comprobación, aplicando las condiciones de contorno (29) facilitadas por el proceso experimental del EWTL.

El proyecto CEDVAL no aporta valores de edad del aire en los puntos de muestreo. El concepto de la edad del aire está relacionado directamente con el valor de los caudales y por tanto de las velocidades del aire. El valor de la edad puntual del aire depende de la trayectoria que sigue desde la admisión por lo que el proceso de validación del modelo de calidad del aire puede ser simplificado comprobando los parámetros de velocidad en un modelo de turbulencia. Este proceso se aplica a dos modelos de turbulencia k-ع: ReNormalization Group (RNG), desarrollado por Yakhot and Orszag (30) y Murakami and Hayashi (29), y Realizable definido por Shih et al. (31), a los que se les aplica el método Enhanced Wall Treatment (EWT) como solución al tratamiento del efecto pared en regímenes turbulentos.

Los resultados obtenidos permiten verificar la correcta configuración CFD de las condiciones de contorno y la calidad del mallado creado, con desviaciones inferiores al margen de error tradicionalmente convenido del $10 \%$ en la región de aire de mayor interés que envuelve al edificio a una distancia inferior a la proporción $2 \cdot H$, obteniéndose una desviación del 4,28\% para el cuartil intermedio.

Se extrae que los resultados de validación para los puntos ubicados a una distancia inferior a $2 \cdot \mathrm{H}$ se encuentran dentro del rango $\pm 7,6 \%$ de los originales (CEDVAL). La media de las velocidades horizontales obtenidas tiene una desviación del $\pm 3,0 \%$.

En la segunda fase de validación se emplea como referencia el modelo B1-2 del proyecto CEDVAL a escala 1/200. Se trata de una matriz de 56 edificios de planta cuadrada «en anillo». Cada lateral del anillo mide $250 \mathrm{~mm}$ con una altura de $60 \mathrm{~mm}$ con un patio en su interior de planta cuadrada que recorre la totalidad de la altura del edificio y cuyos lados tienen una dimensión de $130 \mathrm{~mm}$.

El modelo de referencia para la validación en túnel del viento proporcionado es simulado bajo distintos modelos de viscosi- 
dad. Se relaciona la precisión de los resultados obtenidos con respecto a los resultados aportados por los ensayos realizados en más de 650 puntos de comprobación (Tabla 2).

Tabla 2. Precisión y frecuencia de los resultados del modelo B1-2 (segunda fase de validación).

\begin{tabular}{|c|c|c|c|}
\hline Metodo CFD & $\begin{array}{c}\text { RNG-second } \\
\text { SWF }\end{array}$ & $\begin{array}{c}\text { RNG-second } \\
\text { EWT }\end{array}$ & $\begin{array}{c}\text { Realizable } \\
\text { EWT }\end{array}$ \\
\hline Velocidad abs & $\pm 4,57 \%$ & $\pm 11,19 \%$ & $\pm 3,68 \%$ \\
\hline Velocidad X & $\pm \mathbf{3 , 1 2} \%$ & $\pm \mathbf{3 , 1 2} \%$ & $\pm \mathbf{2 , 0 1 \%}$ \\
\hline Velocidad Y & $\pm 7,35 \%$ & $\pm 7,35 \%$ & $\pm 12,46 \%$ \\
\hline Velocidad Z & $\pm 9,34 \%$ & $\pm 9,35 \%$ & $\pm 4,23 \%$ \\
\hline \multicolumn{2}{|c|}{ Frecuencia de resultados para velocidad en eje $\ll \mathbf{~} \%$} \\
\hline$<\mathbf{5} \%$ & $15,84 \%$ & $2,92 \%$ & $12,43 \%$ \\
\hline $\mathbf{5 - 1 0} \%$ & $29,90 \%$ & $3,57 \%$ & $31,07 \%$ \\
\hline $\mathbf{1 0 - 2 0} \%$ & $22,57 \%$ & $8,12 \%$ & $23,82 \%$ \\
\hline$>\mathbf{2 0} \%$ & $31,69 \%$ & $85,39 \%$ & $32,69 \%$ \\
\hline
\end{tabular}

Se estudia la frecuencia del número de puntos de muestreo cuyos resultados tienen una mayor precisión, suprimidos los valores extremos sobre un percentil del $5 \%$. Se consigue una desviación inferior al $4 \%$ en los resultados de las velocidades axiles. La precisión media de los resultados de la magnitud de la velocidad del viento es superior al $95 \%$ en modelos de simulación de turbulencia «Realizable».

\section{DEFINICIÓN Y SIMULACIÓN DE CASOS}

Este estudio pretende analizar las variables dimensionales del patio para tener un modelo comparativo para el análisis de la idoneidad del diseño de estos espacios exteriores con independencia de las variables externas.

Se plantean edificios aislados en entornos urbanos de muy baja rugosidad con patio central de dimensiones que van desde los dos metros hasta los seis metros de ancho $(W)$ o largo $(L)$ y desde los seis metros hasta los 42 metros de altura $(H)$ (hasta baja+13). El espesor de la construcción $\left(c_{r}\right)$ definida entre el volumen de aire de patio y los límites exteriores del edificio (10 metros) se toma como la longitud de referencia constante para la definición del dominio de control que envuelve al edificio. Así se simulan 175 casos con los mismos criterios de diseño del modelo CFD según las condiciones de contorno (Tabla 3) (32) cuyas dimensiones del edificio varían dependientemente de las dimensiones del patio (Figura 3). El mallado hexaédrico del modelo tridimensional se ha diseñado en base a la capacidad computacional para cada modelo.

Como la eficiencia isoterma del patio (sin afección de movimientos convectivos o efecto de flotabilidad) depende principalmente de la velocidad de la corriente libre, como es lógico, la situación más desfavorable se produce en ausencia de viento $\left(\varepsilon^{\mathrm{a}}=0 \%\right)$. Esta hipótesis aconseja buscar un valor ideal de baja velocidad del viento que induzca la circulación de aire en el interior del patio y que al mismo tiempo sea frecuente en las ubicaciones seleccionadas. Los resultados de medición de la velocidad media en España, publicados por el Centro Nacional de Energías Renovables (CENER) (33), dan un valor medio aproximado de $3 \mathrm{~m} / \mathrm{s}$. Asimismo, el Instituto para la Diversificación y Ahorro de Energía (IDAE) (http:// atlaseolico.idae.es/) pone a disposición mapas eólicos en los que se dan valores medios entre los 2 y los $4 \mathrm{~m} / \mathrm{s}$ a una altura de $80 \mathrm{~m}$ para las ubicaciones indicadas. Se establece una velocidad de referencia de $3 \mathrm{~m} / \mathrm{s}$ a la altura de referencia de $100 \mathrm{~m}$ (Tabla 3).

Los casos han sido simulados mediante métodos de cálculo numérico CFD con la herramienta ANSYS Fluent $15.0^{\odot}$, se-

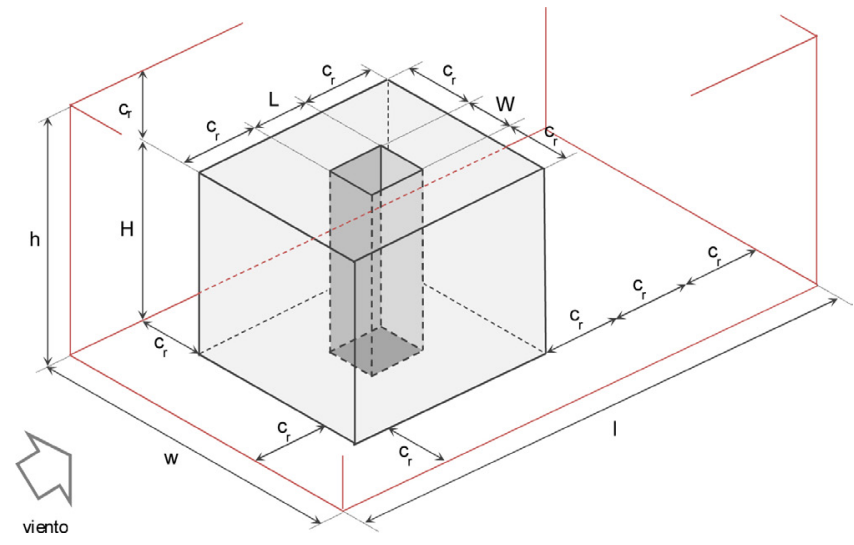

Figura 3. Determinación de las dimensiones del dominio de control según patio.

Tabla 3. Condiciones y criterios de diseño del modelo simulado.

\begin{tabular}{|c|c|c|c|}
\hline \multicolumn{2}{|c|}{ Criterios del modelo CFD } & \multicolumn{2}{|c|}{ Pre-procesado - Mallado } \\
\hline Longitud dominio & $20 \cdot \mathrm{cr}$ & Número de celdas & 1-3 millones \\
\hline Ancho dominio & $5 \cdot \mathrm{cr}$ & Proporción máxima & $1 / 4$ \\
\hline Alto dominio & $6 \cdot \mathrm{cr}$ & Máx. ratio «inflation» & $1,08(<8 \%)$ \\
\hline \multicolumn{2}{|l|}{ Malla hexaédrica } & Celda mínima & $0,025 \mathrm{~m}$ \\
\hline \multicolumn{2}{|c|}{ Características del aire (fluido) } & \multicolumn{2}{|c|}{\begin{tabular}{|l} 
Condiciones de contorno de la admisión \\
\end{tabular}} \\
\hline \multirow{2}{*}{ Densidad del aire } & \multirow{2}{*}{$1.204 \mathrm{~kg} / \mathrm{m}^{3}$} & Velocidad de referencia & $3.000 \mathrm{~m} / \mathrm{s}$ \\
\hline & & Altura de referencia & $100.000 \mathrm{~m}$ \\
\hline Temperatura & $293,751 \mathrm{~K}$ & Intensidad de turbulencia & Ecus. [6] y [7] \\
\hline Número de Reynolds & 37250 & Disipación de la turbulencia & Ecus. [8] y [9] \\
\hline Viscosidad cinética & $1.515 \mathrm{E}-05 \mathrm{~m}^{2} / \mathrm{s}$ & Altura de turbulencia & $64.000 \mathrm{~m}$ \\
\hline Viscosidad dinámica & $1.825 \mathrm{E}-06 \mathrm{~N} \cdot \mathrm{s} / \mathrm{m}^{2}$ & Constante de von Karman & 0,410 \\
\hline \multicolumn{2}{|c|}{ Condiciones pared } & \multicolumn{2}{|c|}{ Condiciones de contorno isotermas para el suelo } \\
\hline Altura de rugosidad & $0,000 \mathrm{~m}$ & Ley exponencial & 0,220 \\
\hline Altura de desplazamiento & $0,000 \mathrm{~m}$ & Velocidad de fricción & $0,178 \mathrm{~m} / \mathrm{s}$ \\
\hline Temperatura & $293.751 \mathrm{~K}$ & Altura de rugosidad & $0,100 \mathrm{~m}$ \\
\hline \multicolumn{2}{|c|}{ Paredes superior y laterales definidas según límites en simetría } & Altura de desplazamiento & $0,000 \mathrm{~m}$ \\
\hline
\end{tabular}


gún las ecuaciones que definen los perfiles verticales de velocidad y los perfiles de energía y disipación turbulentas del viento [5] [6] [7] [8] [9] (34).

$$
\begin{gathered}
U=\frac{U^{*}}{K} \cdot \operatorname{Ln}\left(\frac{Z-d}{Z_{\mathrm{o}}}\right) \\
k_{n w}=\frac{0,045 \cdot \rho \cdot U_{m} \cdot(\alpha+1)^{2}}{2\left(\frac{U_{m} \cdot h_{t} \cdot(\alpha+1)}{\mu}\right)^{0,25}} \\
k=k_{n w}+\frac{Z}{h_{t}} \cdot\left(0,002 \cdot\left(U_{m} \cdot(\alpha+1)\right)^{2}-k_{n w}\right) \\
\varepsilon=\frac{C_{\mu}^{0,75} \cdot k^{1,5}}{\mathrm{~K} \cdot z} \text { si } z \leq 0,085 h_{t} \\
\varepsilon=\frac{C_{\mu}^{0.75} \cdot k^{1.5}}{0,085 \cdot h_{t}} \text { si } z>0,085 h_{t}
\end{gathered}
$$

Siendo: $U$ la velocidad del viento $(\mathrm{m} / \mathrm{s})$ a la altura $z(\mathrm{~m}) ; d$ la altura de desplazamiento para el perfil del viento (m); $k_{n w}$ la energía turbulenta próxima a los límites del modelo por el efecto pared $\left(\mathrm{m}^{2} / \mathrm{s}^{2}\right) ; \rho$ la densidad del aire $\left(\mathrm{kg} / \mathrm{m}^{3}\right)$ a una temperatura de 293,75 K; $U_{m}$ la velocidad media del perfil exponencial del viento $(\mathrm{m} / \mathrm{s}) ; \alpha$ el exponente adimensional del perfil suburbano del viento; $h_{t}$ la altura del modelo (m) y; $\mu$ la viscosidad dinámica del aire $\left(\mu=1.825 \cdot 10-5 \mathrm{~N} \cdot \mathrm{s} / \mathrm{m}^{2}\right.$ a la temperatura de 293,75 K); $k$ la energía cinética turbulenta $\left(\mathrm{m}^{2} / \mathrm{s}^{2}\right) ; \varepsilon$ el índice de disipación de la turbulencia $\left(\mathrm{m}^{2} / \mathrm{s}^{3}\right) \mathrm{y}$; $C_{\mu}$ constante empírica (determinada por Launder et al. (1974) (35) con un valor aproximado de 0,09).

\section{DISCUSIÓN DE RESULTADOS}

La eficiencia de la ventilación en los patios ha sido analizada por dos métodos complementarios empleándose la edad del aire simulada mediante CFD.

El primer método consiste en asignar como dominio de control el volumen de aire circundante del edificio incluyendo el aire en el patio. De este modo obtenemos la eficiencia de la renovación en distintas regiones del modelo según el comportamiento del aire en el exterior, mediante la división del modelo en volúmenes de interés menores (36). Los resultados obtenidos muestran cómo afecta el patio a la eficiencia en torno al edificio y viceversa. El dominio de control resultante de $72 \mathrm{~m}$ de largo, $52 \mathrm{~m}$ de ancho y $52 \mathrm{~m}$ de alto $(l \times w \times h)$ es dimensionado para cubrir el edificio con un patio cuadrado de hasta $12 \mathrm{~m}$ de lado y $42 \mathrm{~m}$ de altura.

En los resultados alcanzados se comprueba que el ancho del patio apenas supone variación reseñable en la eficiencia y en el consecuente reparto del aire «limpio» en su interior, por lo que a modo ejemplo se expone (Tabla 4) lo que teóricamente cabe preverse, como:

- que cuanto mayor es el volumen del patio, el tiempo mínimo de renovación del dominio de control se reduce debido a la reducción de la sección eficaz para el flujo de aire de forma contraria a lo que sucede con la edad media en el dominio. El menor de los patios aporta una eficiencia
(47,71\%) próxima al modelo mezcla $(\approx 50 \%)$ en el dominio de control. La edad media en el dominio crece por influencia del estancamiento del aire en el interior del patio, lo que lleva a que su eficiencia descienda hasta un $7,52 \%$ en el peor de los casos;

- que a mayor altura del patio, su eficiencia desciende de forma no lineal. Sin embargo, la eficiencia aumenta al aumentarse la longitud y la anchura del patio respectivamente. Esto es debido a la capacidad del aire en su acceso al interior del patio. La mejora se hace más notable cuanto mayor es su longitud debido a la tendencia del aire a retornar a las capas inferiores una vez disipada la energía turbulenta vertical generada en la cornisa expuesta del edificio, lo cual es debido principalmente a la velocidad del viento. El aire desprendido de la cubierta accede al patio cuando es arrastrado por debajo de la cota de coronación del mismo a la distancia del despegue en el que se encuentra con el vacío (Figura 4), comprendida entre el espesor del edificio y la longitud del patio y;

- que el mayor estancamiento se produce en las cotas inferiores del patio. Esto es debido a la formación de vórtices que reducen la energía dinámica en la embocadura del patio taponando la salida y reduciendo la capacidad de desplazamiento de las partículas de las cotas inferiores (Figura 5). Analizándose individualmente cada patio, dividida su altura en tres partes obtenemos que el tercio intermedio presenta una eficiencia media del $91 \%$ de la eficiencia total del patio, mientras que el tercio inferior presenta una reducción media del $50 \%$, valores que oscilan entre el $27 \%$ y el $151 \%$, y entre el $15 \%$ y el $75 \%$ respectivamente.

El segundo método corrobora lo analizado mediante el método de cálculo por la envolvente. Se analiza el comportamiento del aire contenido en el patio por influencia de la velocidad vertical y la turbulencia en la embocadura del patio. Esto permite representar gráficamente (Figura 6) la repercusión que tienen las dimensiones del patio sobre el proceso de ventilación exterior y la calidad del aire a ser suministrado al interior de los habitáculos. Los valores de la eficiencia intermedias entre los casos analizados se obtienen de forma aproximada mediante la interpolación geométrica bidimensional. Se verifica que las variaciones de las eficiencias en los distintos casos analizados siguen un patrón dependiente principalmente de la dimensión longitudinal.

Por el propio concepto y diseño de patio, son espacios exteriores que ven reducida considerablemente la calidad de su aire con respecto a los cánones de salubridad e higiene aceptados. Se obtienen los mejores resultados de eficiencia cuanto más bajo es el patio y más largo en la dirección predominante del viento.

\subsection{Aplicación de resultados}

Los resultados obtenidos verifican las hipótesis tomadas y cuantifican la influencia de las dimensiones del patio sobre la calidad del aire a ser suministrado al interior de las viviendas. El objetivo del presente trabajo radica en la aplicación del concepto de la eficiencia de la ventilación exterior para analizar de un modo objetivo las principales normas sobre patios en España. Se presenta el cuadro comparativo en el 


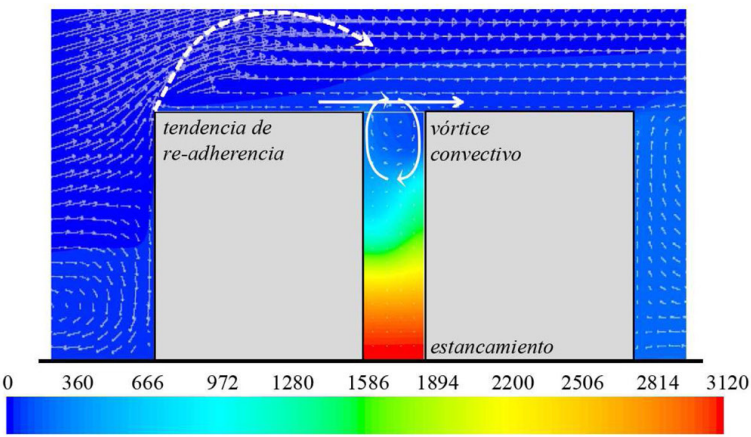

(a) $3 \times 6 \times 12$

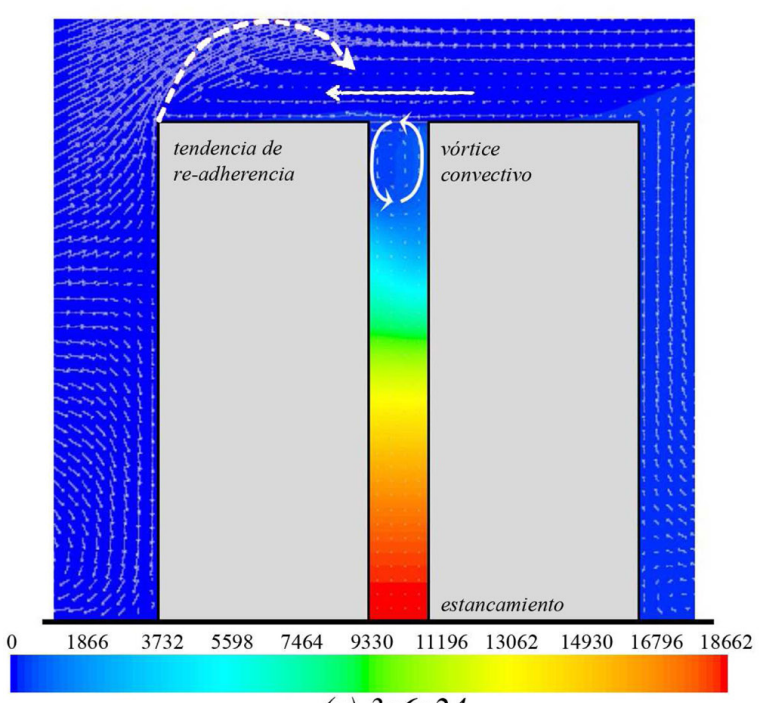

(c) $3 \times 6 \times 24$

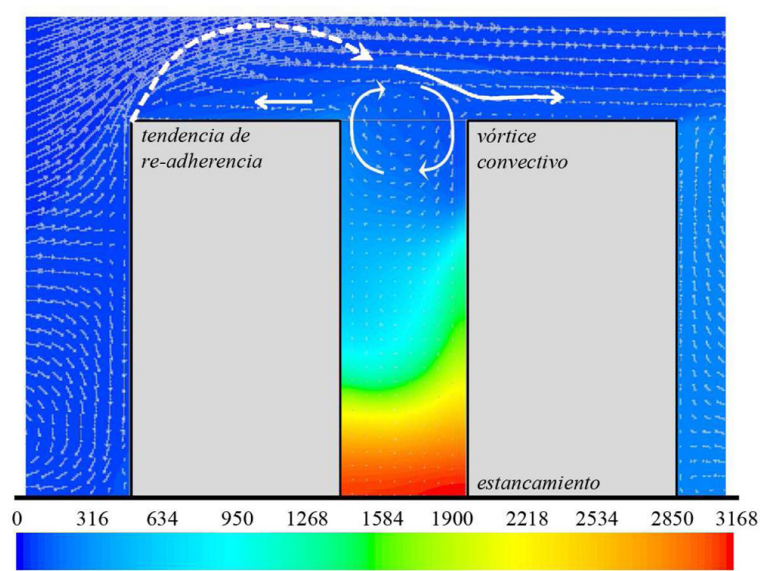

(b) $6 \times 6 \times 18$

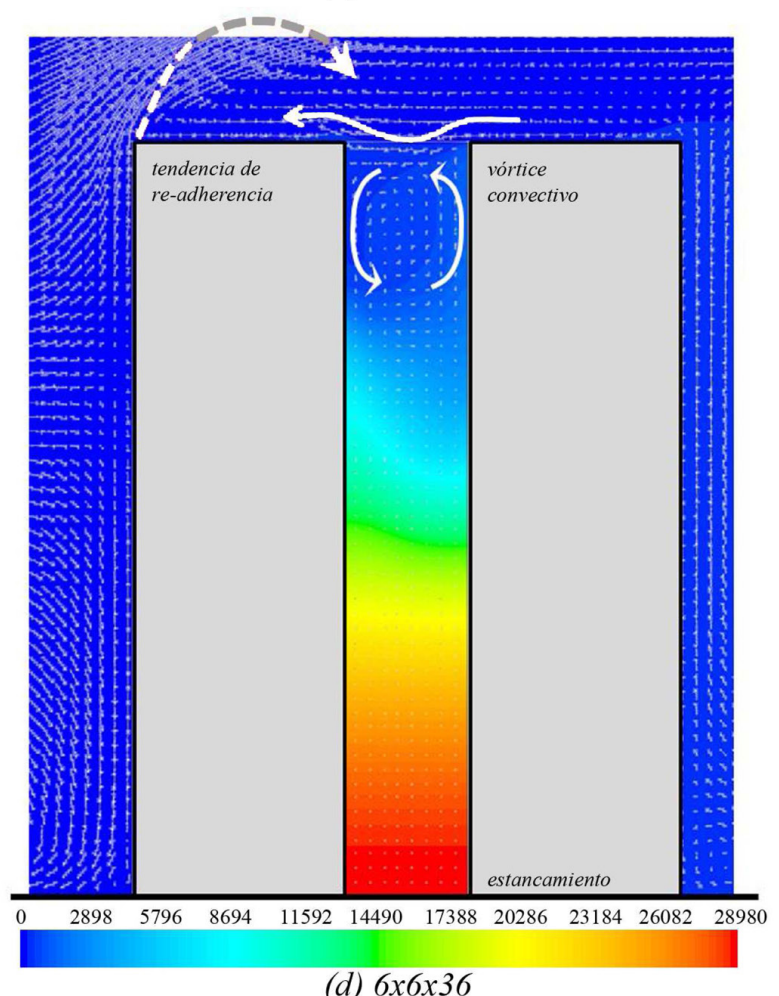

(d) $6 \times 6 \times 36$

Figura 4. Influencia del comportamiento en la distribución de la edad del aire puntual (s).

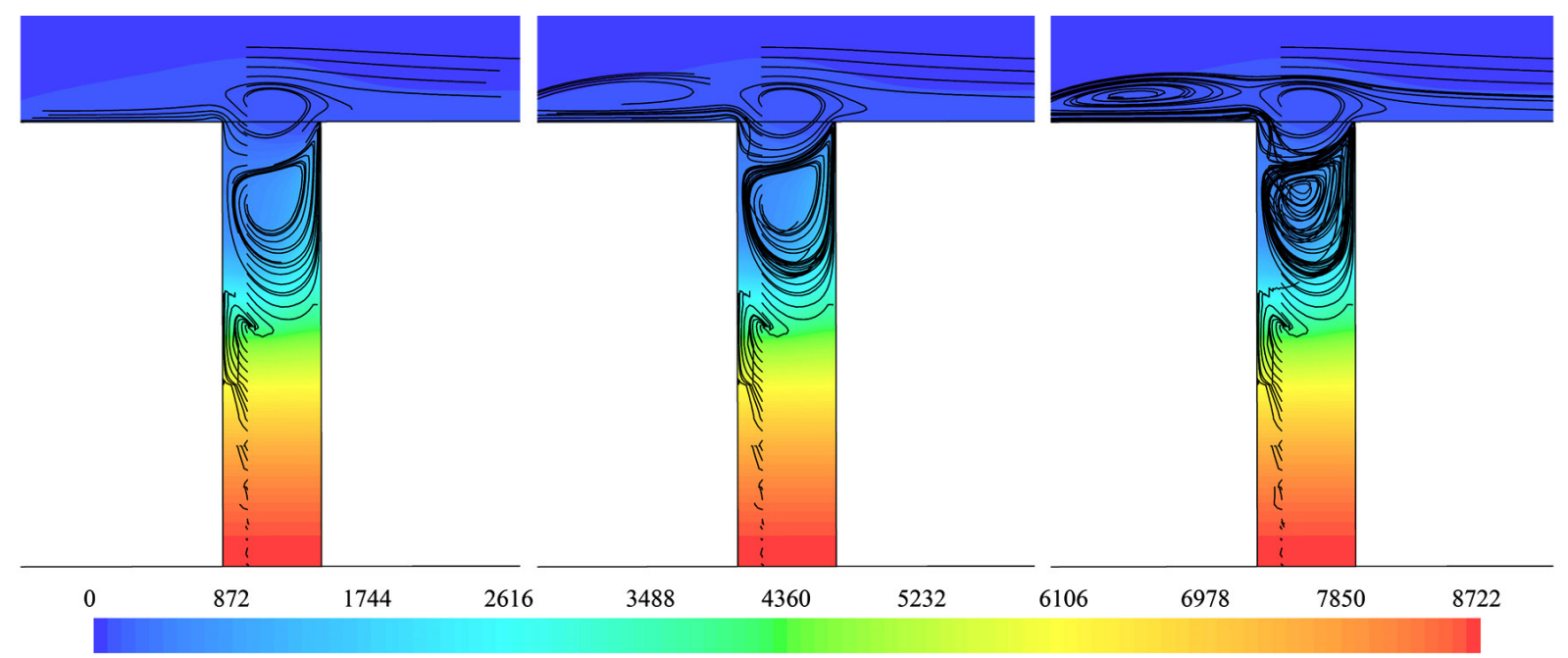

Figura 5. Evolución de la trayectoria seguida por una columna de aire a L/4 del cerramiento a sotavento del patio $(4 \times 3 \times 18)$ y su impacto en la edad del aire $(\mathrm{s})$. 

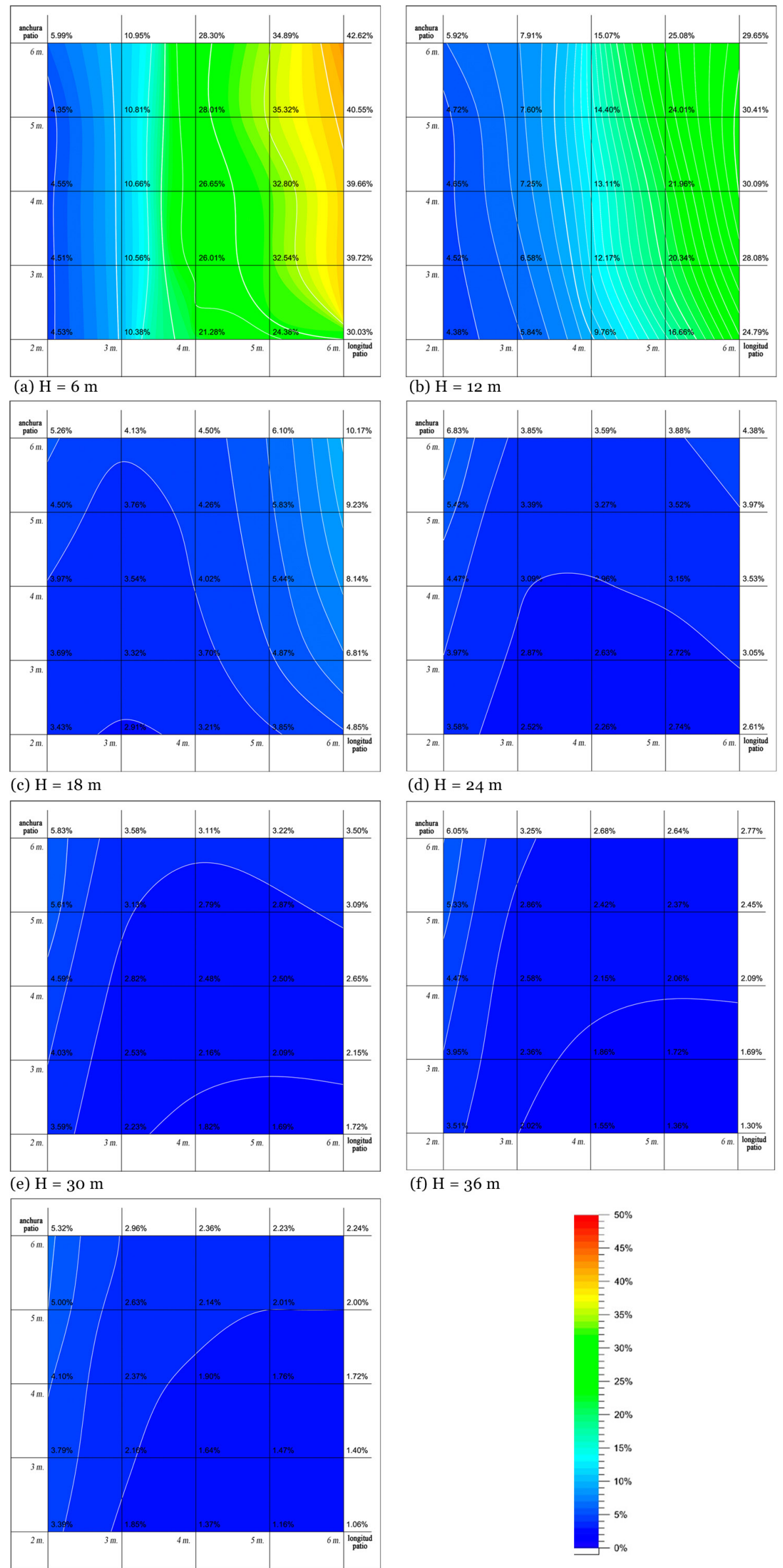

(d) $\mathrm{H}=24 \mathrm{~m}$

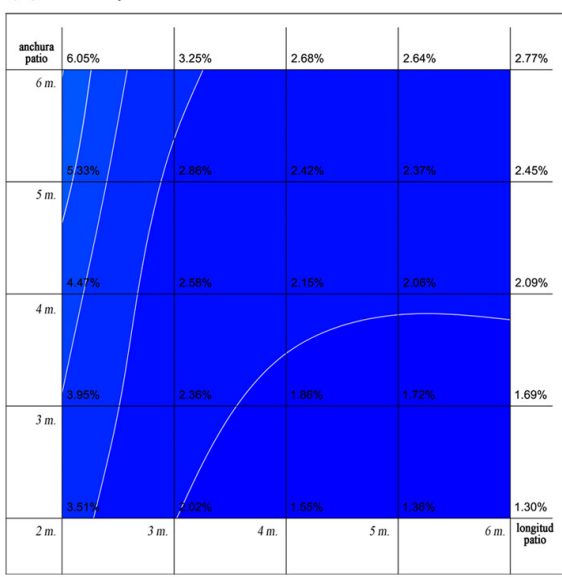

(f) $\mathrm{H}=36 \mathrm{~m}$

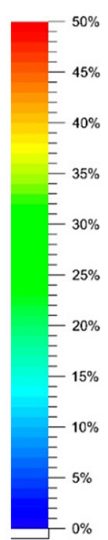

(g) $\mathrm{H}=42 \mathrm{~m}$

Figura 6. Eficiencias relativas de renovación del aire en los patios analizados (\%). 
Tabla 4. Ejemplo de los resultados numéricos de las edades medias y las eficiencias de los casos analizados: patios de ancho fijo de $4 \mathrm{~m}$.

\begin{tabular}{|c|c|c|c|c|c|c|c|c|}
\hline \multirow{2}{*}{ patio } & \multirow{2}{*}{$\begin{array}{c}\text { tiempo } \\
\text { mínimo }\end{array}$} & \multicolumn{2}{|c|}{$\begin{array}{l}\text { edad media del volumen } \\
\qquad\langle\bar{\tau}\rangle(\mathrm{s})\end{array}$} & \multicolumn{5}{|c|}{$\begin{array}{l}\text { eficiencia } \\
\boldsymbol{\varepsilon}^{a}(\%)\end{array}$} \\
\hline & & $\begin{array}{l}\text { dominio } \\
\text { control }\end{array}$ & patio & $\begin{array}{l}\text { dominio } \\
\text { control }\end{array}$ & patio & $\begin{array}{l}\text { tercio } \\
\text { superior }\end{array}$ & $\begin{array}{l}\text { tercio } \\
\text { medio }\end{array}$ & $\begin{array}{l}\text { tercio } \\
\text { inferior }\end{array}$ \\
\hline $2 \times 4 \times 6$ & 61,17 & 65,0 & 702,8 & $47,03 \%$ & $4,35 \%$ & $11,06 \%$ & $3,34 \%$ & $2,54 \%$ \\
\hline $2 \times 4 \times 12$ & 60,15 & 57,3 & 3077,1 & $44,68 \%$ & $0,98 \%$ & $3,45 \%$ & $0,89 \%$ & $0,61 \%$ \\
\hline $2 \times 4 \times 18$ & 59,14 & 84,1 & 7881,8 & $35,16 \%$ & $\mathbf{0 , 3 8 \%}$ & $1,21 \%$ & $0,33 \%$ & $0,24 \%$ \\
\hline $2 \times 4 \times 24$ & 58,13 & 108,3 & 14278,6 & $26,84 \%$ & $0,20 \%$ & $0,64 \%$ & $0,18 \%$ & $0,13 \%$ \\
\hline $\mathbf{2} \times 4 \times 30$ & 57,12 & 140,9 & 23064,5 & $20,28 \%$ & $0,12 \%$ & $0,38 \%$ & $0,11 \%$ & $0,08 \%$ \\
\hline $2 \times 4 \times 36$ & 56,11 & 189,8 & 34392,2 & $14,78 \%$ & $\mathbf{0 , 0 8 \%}$ & $0,24 \%$ & $0,07 \%$ & $0,05 \%$ \\
\hline $2 \times 4 \times 42$ & 55,10 & 239,2 & 46921,0 & $11,52 \%$ & $0,06 \%$ & $0,17 \%$ & $0,05 \%$ & $0,04 \%$ \\
\hline $3 \times 4 \times 6$ & 61,13 & 65,0 & 343,6 & $47,00 \%$ & $8,90 \%$ & $32,65 \%$ & $7,52 \%$ & $4,00 \%$ \\
\hline $3 \times 4 \times 12$ & 60,08 & 66,9 & 1762,1 & $44,93 \%$ & $1,70 \%$ & $7,39 \%$ & $1,74 \%$ & $0,95 \%$ \\
\hline $3 \times 4 \times 18$ & 59,03 & 84,7 & 6035,8 & $34,83 \%$ & $0,49 \%$ & $2,24 \%$ & $0,44 \%$ & $0,29 \%$ \\
\hline $3 \times 4 \times 24$ & 57,98 & 110,8 & 11867,7 & $26,16 \%$ & $0,24 \%$ & $1,01 \%$ & $0,22 \%$ & $0,15 \%$ \\
\hline $3 \times 4 \times 30$ & 56,93 & 147,5 & 19690,1 & $19,30 \%$ & $0,14 \%$ & $0,56 \%$ & $0,13 \%$ & $0,09 \%$ \\
\hline $3 \times 4 \times 36$ & 55,88 & 204,6 & 29956,1 & $13,65 \%$ & $\mathbf{0 , 0 9 \%}$ & $0,33 \%$ & $0,08 \%$ & $0,06 \%$ \\
\hline $3 \times 4 \times 42$ & 54,83 & 272,9 & 42738,1 & $10,05 \%$ & $0,06 \%$ & $0,21 \%$ & $0,06 \%$ & $0,04 \%$ \\
\hline $4 \times 4 \times 6$ & 61,09 & 66,1 & 151,5 & $46,22 \%$ & $20,16 \%$ & $27,10 \%$ & $8,50 \%$ & $4,02 \%$ \\
\hline $4 \times 4 \times 12$ & 60,00 & 68,1 & 921,9 & $44,03 \%$ & $3,25 \%$ & $12,63 \%$ & $3,42 \%$ & $1,90 \%$ \\
\hline $4 \times 4 \times 18$ & 58,91 & 85,0 & 4255,8 & $34,64 \%$ & $0,69 \%$ & $3,88 \%$ & $0,69 \%$ & $0,38 \%$ \\
\hline $4 \times 4 \times 24$ & 57,82 & 112,7 & 9220,7 & $25,64 \%$ & $\mathbf{0 , 3 1} \%$ & $1,80 \%$ & $0,29 \%$ & $0,18 \%$ \\
\hline $4 \times 4 \times 30$ & 56,73 & 152,5 & 15884,8 & $18,60 \%$ & $0,18 \%$ & $0,93 \%$ & $0,16 \%$ & $0,10 \%$ \\
\hline $4 \times 4 \times 36$ & 55,64 & 216,0 & 24788,9 & $12,88 \%$ & $\mathbf{0 , 1 1} \%$ & $0,53 \%$ & $0,10 \%$ & $0,07 \%$ \\
\hline $4 \times 4 \times 42$ & 54,55 & 294,4 & 36037,1 & $9,27 \%$ & $\mathbf{0 , 0 8 \%}$ & $0,32 \%$ & $0,07 \%$ & $0,05 \%$ \\
\hline $5 \times 4 \times 6$ & 61,05 & 66,0 & 128,9 & $46,26 \%$ & $23,68 \%$ & $24,28 \%$ & $6,38 \%$ & $4,77 \%$ \\
\hline $5 \times 4 \times 12$ & 59,92 & 67,1 & 533,0 & $44,62 \%$ & $5,62 \%$ & $15,57 \%$ & $5,74 \%$ & $3,51 \%$ \\
\hline $5 \times 4 \times 18$ & 58,79 & 82,8 & 2655,1 & $35,49 \%$ & $\mathbf{1 , 1 1 \%}$ & $4,94 \%$ & $1,37 \%$ & $0,56 \%$ \\
\hline $5 \times 4 \times 24$ & 57,66 & 109,6 & 7080,0 & $26,30 \%$ & $0,41 \%$ & $3,05 \%$ & $0,40 \%$ & $0,22 \%$ \\
\hline $5 \times 4 \times 30$ & 56,54 & 146,6 & 12473,9 & $19,28 \%$ & $0,23 \%$ & $1,51 \%$ & $0,22 \%$ & $0,13 \%$ \\
\hline $5 \times 4 \times 36$ & 55,41 & 206,7 & 20023,0 & $13,40 \%$ & $0,14 \%$ & $0,86 \%$ & $0,13 \%$ & $0,08 \%$ \\
\hline $5 \times 4 \times 42$ & 54,28 & 282,2 & 29859,4 & $9,62 \%$ & $\mathbf{0 , 0 9 \%}$ & $0,51 \%$ & $0,08 \%$ & $0,05 \%$ \\
\hline $6 \times 4 \times 6$ & 61,01 & 66,0 & 100,7 & $46,20 \%$ & $30,31 \%$ & $18,99 \%$ & $18,80 \%$ & $5,95 \%$ \\
\hline $6 \times 4 \times 12$ & 59,84 & 66,0 & 233,8 & $45,35 \%$ & $12,80 \%$ & $17,74 \%$ & $9,91 \%$ & $5,13 \%$ \\
\hline $6 \times 4 \times 18$ & 58,68 & 80,4 & 1555,1 & $36,50 \%$ & $1,89 \%$ & $7,32 \%$ & $2,37 \%$ & $0,97 \%$ \\
\hline $6 \times 4 \times 24$ & 57,51 & 108,4 & 5377,4 & $26,54 \%$ & $\mathbf{0 , 5 3 \%}$ & $3,93 \%$ & $0,60 \%$ & $0,27 \%$ \\
\hline $6 \times 4 \times 30$ & 56,34 & 147,0 & 9821,2 & $19,16 \%$ & $\mathbf{0 , 2 9 \%}$ & $2,33 \%$ & $0,29 \%$ & $0,15 \%$ \\
\hline $6 \times 4 \times 36$ & 55,18 & 212,5 & 16239,3 & $12,98 \%$ & $0,17 \%$ & $1,24 \%$ & $0,17 \%$ & $0,09 \%$ \\
\hline $6 \times 4 \times 42$ & 54,01 & 298,7 & 24817,2 & $9,04 \%$ & $0,11 \%$ & $0,76 \%$ & $0,10 \%$ & $0,06 \%$ \\
\hline
\end{tabular}


Tabla 5. Aplicación del concepto de eficiencia para la evaluación de los patios en España.

\begin{tabular}{|c|c|c|c|c|c|c|c|c|c|c|c|c|c|c|c|c|c|c|c|c|c|c|c|c|c|c|c|c|c|c|c|c|c|c|c|}
\hline & & N & $\begin{array}{c}\infty \\
\boldsymbol{N} \\
\mathbf{x} \\
\boldsymbol{N} \\
x \\
\boldsymbol{N}\end{array}$ & $\begin{array}{l}\vec{N} \\
\mathbf{X} \\
\mathbf{N} \\
\times \\
\mathbf{N} \\
\end{array}$ & \begin{tabular}{|c|c}
0 \\
$\dot{m}$ \\
$x$ \\
$N$ \\
$x$ \\
$\boldsymbol{N}$ \\
\end{tabular} & $\begin{array}{l}0 \\
m \\
x \\
N \\
\times \\
N \\
N\end{array}$ & $\begin{array}{l}\vec{y} \\
x \\
\ddot{x} \\
x\end{array}$ & $\begin{array}{l}0 \\
x \\
m\end{array}$ & & $\begin{array}{l}\stackrel{\infty}{\vec{x}} \\
\text { m }\end{array}$ & $\begin{array}{l}\text { N } \\
x \\
\text { m }\end{array}$ & $\begin{array}{l}0 \\
\dot{m} \\
x \\
m \\
x \\
\boldsymbol{N}\end{array}$ & $\begin{array}{l}0 \\
m \\
x \\
m \\
x \\
v\end{array}$ & $\begin{array}{l}\text { Vy } \\
x \\
\mathscr{x} \\
x \\
\text { N }\end{array}$ & $\begin{array}{l}0 \\
\times \\
+ \\
\times \\
\text { N } \\
\end{array}$ & N & e & $\begin{array}{l}4 \\
4 \\
4\end{array}$ & & $\begin{array}{l}\dot{m} \\
x \\
\dot{x}\end{array}$ & $\begin{array}{l}F \\
\dot{x} \\
\dot{y}\end{array}$ & $\begin{array}{l}0 \\
x \\
10\end{array}$ & $\begin{array}{l}\text { N } \\
\times \\
10 \\
\times \\
\text { N } \\
\end{array}$ & $\stackrel{\infty}{=}$ & $\begin{array}{c}x \\
x \\
4 \\
4\end{array}$ & م: & b & $\begin{array}{l}7 \\
\times \\
x\end{array}$ & $\begin{array}{l}0 \\
x \\
b \\
x \\
\boldsymbol{N}\end{array}$ & $\begin{array}{l}\mathbf{N} \\
\times \\
6 \\
\times \\
\text { N }\end{array}$ & $\begin{array}{l}\infty \\
\vec{x} \\
\times \\
0 \\
x \\
N\end{array}$ & $\begin{array}{l}\mathbb{N} \\
\times \\
\times \\
0 \\
\times \\
\boldsymbol{N}\end{array}$ & $\begin{array}{l}x \\
b \\
x \\
\hat{N}\end{array}$ & $\begin{array}{l}0 \\
\dot{m} \\
x \\
6 \\
x \\
\text { N }\end{array}$ & \\
\hline & & - & - & - & 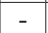 & - & - & - & - & - & - & - & - & - & - & . & & & & - & - & - & - & - & - & 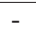 & - & - & - & - & & - & - & & \\
\hline & & - & - & - & - & - & - & & 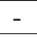 & - & - & - & - & - & - & - & & & & - & - & - & - & & & & & - & - & - & - & - & - & & \\
\hline & & - & - & - & - & - & 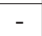 & & & - & - & - & - & - & - & & & & & - & - & - & - & & & & & & & - & & & & & \\
\hline & & & - & - & - & - & - & & 도 & - & - & - & - & - & $\square$ & & & & & - & - & $\square$ & П & & & & & - & 0 & 0 & - & - & - & - & \\
\hline & & - & - & - & - & - & - & , & - & - & - & - & - & - & 口 & 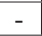 & . & & & - & - & 足 & П & & & - & - & - & $\circ$ & $\circ$ & - & - & - & - & \\
\hline & & - & - & - & 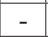 & - & - & 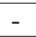 & - & - & - & - & - & - & - & - & & & & - & - & - & - & - & - & - & - & - & - & - & - & - & - & - & \\
\hline & & - & - & - & - & - & - & $\square$ & - & - & - & - & - & - & $\square$ & - & - & - & & - & - & 口 & - & - & - & - & - & - & $\circ$ & - & - & - & - & - & \\
\hline & 다 & 마 & - & - & - & - & - & 드. & ㄷ & - & - & - & - & - & 口 & 도 & - & - & & - & - & $\square$ & 口 & - & - & - & - & - & $\circ$ & $\circ$ & - & - & - & - & \\
\hline$\dot{G}-\mathbf{B} \mathbf{1}$ & & - & - & - & - & - & - & & - & - & - & - & - & - & - & & & & & - & - & - & - & - & - & & & & - & - & - & - & - & - & \\
\hline
\end{tabular}

\begin{tabular}{|c|c|c|c|c|c|c|c|c|c|c|c|c|c|c|c|c|c|c|c|c|c|c|c|c|c|c|c|c|c|c|c|c|c|c|c|}
\hline & & $\begin{array}{l} \\
x \\
x \\
-\infty\end{array}$ & $\begin{array}{l}\mathbb{N} \\
\mathbf{x} \\
\boldsymbol{m}\end{array}$ & 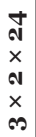 & $\begin{array}{l}x \\
\boldsymbol{N} \\
x \\
\boldsymbol{r}\end{array}$ & $\begin{array}{l}0 \\
m \\
\times \\
N \\
\times \\
\infty\end{array}$ & $\begin{array}{l}\mathcal{Y} \\
x \\
x \\
N \\
x \\
\text { O }\end{array}$ & $\begin{array}{l}0 \\
x \\
m \\
x \\
m\end{array}$ & $\begin{array}{l}m \\
x \\
m\end{array}$ & $\infty$ & \% & $\begin{array}{l}0 \\
x \\
m\end{array}$ & $\begin{array}{c}0 \\
0 \\
x \\
m \\
x \\
x \\
m\end{array}$ & $\mid \begin{array}{c}\tilde{y} \\
x \\
\tilde{x} \\
\tilde{x} \\
\tilde{n}\end{array}$ & $\begin{array}{l}0 \\
x \\
\dot{r} \\
x \\
\infty\end{array}$ & $\begin{array}{l}\mathfrak{N} \\
\times \\
\dot{+} \\
\times \\
-\infty\end{array}$ & $\begin{array}{l}\infty \\
\vec{x} \\
\times \\
\dot{+} \\
\times \\
\infty\end{array}$ & $\begin{array}{l}x \\
\vec{r} \\
x \\
m\end{array}$ & $\begin{array}{c}\dot{\rho} \\
x \\
x \\
\dot{+} \\
x \\
m\end{array}$ & $\begin{array}{c}0 \\
\infty \\
x \\
\dot{+} \\
x \\
\infty\end{array}$ & $\begin{array}{c}\text { Iv } \\
x \\
x \\
\dot{y} \\
x \\
\text { m }\end{array}$ & $\begin{array}{c}0 \\
x \\
10 \\
x \\
0\end{array}$ & 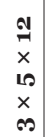 & $\begin{array}{c}\infty \\
\vec{x} \\
10 \\
\times \\
\infty\end{array}$ & $\begin{array}{l}\vec{N} \\
\times \\
10 \\
\times \\
\infty\end{array}$ & $\begin{array}{l}0 \\
\dot{x} \\
x \\
10 \\
x \\
m\end{array}$ & $\begin{array}{l}x \\
10 \\
x \\
n\end{array}$ & $\begin{array}{l}x \\
10 \\
x \\
\infty \\
\infty\end{array}$ & $\begin{array}{l}x \\
0 \\
x \\
x \\
m\end{array}$ & 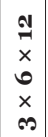 & $\begin{array}{l}\infty \\
\vec{x} \\
0 \\
\times \\
x \\
\infty\end{array}$ & $\begin{array}{l}\mathbb{N} \\
\times \\
6 \\
\times \\
\infty\end{array}$ & $\begin{array}{l}0 \\
\dot{m} \\
x \\
6 \\
x \\
\infty\end{array}$ & & \\
\hline & & & & & - & -1 & -1 & 0 & 0 & 口 & ᄆ & 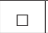 & $\square$ & \begin{tabular}{|l|}
$\square$ \\
\end{tabular} & $\circ$ & $\circ$ & $\square$ & 口 & $\square$ & - & $\square$ & 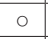 & 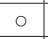 & 口 & \begin{tabular}{l|l} 
\\
\end{tabular} & \begin{tabular}{l|l} 
\\
\end{tabular} & \begin{tabular}{|l|}
$\square$ \\
\end{tabular} & $\square$ & 0 & 0 & $\square$ & $\square$ & $\square$ & $\square$ & \\
\hline & & & & & . & 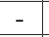 & 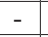 & $c$ & & & & & - & & & & & & & & - & $c$ & & & & & 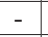 & & & & & & & & \\
\hline & & & - & - & - & - & - & c & $c$ & [ & $\square$ & ㅁ & $\square$ & 口 & $\circ$ & & 다 & 드 & $\square$ & $\square$ & $\square$ & $\circ$ & $\circ$ & & $\square$ & $\square$ & 口 & ㅁ & $\circ$ & $\circ$ & & $\square$ & 므 & 口 & \\
\hline & & & & & - & - & - & $c$ & & & 5 & $\square$ & 口 & $\square$ & $\circ$ & & & & ㅁ & 다 & ㅁ & $\circ$ & $\circ$ & & 므 & 마 & 므 & & & $\circ$ & & & $\square$ & & \\
\hline & & & & & - & - & - & c & $c$ & & & - & - & - & $\circ$ & $\circ$ & & & & & . & 0 & & & & & & & & $\circ$ & & & & & \\
\hline & & & & & - & - & - & - & - & - & . & - & - & - & $\circ$ & $\circ$ & ㅁ & 口 & ㅁ & $\square$ & $\square$ & $\circ$ & o & & 口 & 口 & $\square$ & П & ه & $\circ$ & & $\square$ & $\square$ & & \\
\hline & & & - & - & - & - & - & 0 & $\circ$ & - & - & - & 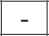 & - & $\circ$ & $\circ$ & - & - & - & - & 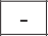 & + & 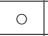 & - & 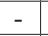 & - & - & - & $\circ$ & $\circ$ & & - & - & & \\
\hline & $\stackrel{0}{2}$ & $\circ$ & 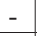 & - & - & - & - & $\circ$ & $\circ$ & $\square$ & ᄃ & 口 & $\square$ & $\square$ & $\circ$ & $\circ$ & ㅁ & $\square$ & $\square$ & $\square$ & $\square$ & $\circ$ & $\circ$ & $\square$ & $\square$ & $\square$ & $\square$ & $\square$ & $\circ$ & $\circ$ & $\square$ & $\square$ & $\square$ & $\square$ & \\
\hline $8-1$ & & & & & - & - & - & $\circ$ & 0 & & & - & - & - & 0 & & ㅁ & & - & & - & $\circ$ & $\circ$ & & & & & & & $\circ$ & & & - & & \\
\hline
\end{tabular}

\begin{tabular}{|c|c|c|c|c|c|c|c|c|c|c|c|c|c|c|c|c|c|c|c|c|c|c|c|c|c|c|c|c|c|c|c|c|c|c|c|}
\hline & & & $\begin{array}{l}\ddot{x} \\
\dot{t}\end{array}$ & t & $\begin{array}{l}x \\
\dot{t}\end{array}$ & $\begin{array}{l} \\
m \\
x \\
N \\
x \\
+ \\
\end{array}$ & $\begin{array}{l}\dot{\vec{\sigma}} \\
\times \\
\vec{N} \\
\dot{x} \\
\vec{\nabla} \\
\end{array}$ & $\begin{array}{l}0 \\
x \\
\ddot{x} \\
x \\
\overrightarrow{+}\end{array}$ & $\begin{array}{l}0 \\
x \\
+\end{array}$ & $\begin{array}{l}m \\
x \\
+\end{array}$ & $\hat{\nabla}$ & & $\begin{array}{l}0 \\
m \\
x \\
m \\
x \\
+\end{array}$ & $\begin{array}{l}\text { Vै } \\
\times \\
x \\
0 \\
\times \\
+ \\
\end{array}$ & $\begin{array}{l}0 \\
x \\
+ \\
\times \\
+ \\
\end{array}$ & $\begin{array}{l}\vec{\nabla} \\
\times \\
\vec{\nabla}\end{array}$ & $\begin{array}{l}\infty \\
\vec{x} \\
x \\
+ \\
x \\
+\end{array}$ & $\begin{array}{l}x \\
+\end{array}$ & $\begin{array}{l}x \\
\dot{y}\end{array}$ & $\begin{array}{l}0 \\
\dot{m} \\
x \\
+ \\
\times \\
+ \\
\end{array}$ & $\begin{array}{l}\text { Iै } \\
x \\
\dot{y} \\
\times \\
\dot{y}\end{array}$ & $\begin{array}{l}0 \\
x \\
10 \\
x \\
+ \\
\end{array}$ & $\begin{array}{l}\text { I } \\
\vec{x} \\
10 \\
x \\
+ \\
\end{array}$ & $\begin{array}{l}\infty \\
\vec{x} \\
x \\
10 \\
\times \\
+\end{array}$ & $\begin{array}{l}\mathbf{N} \\
\times \\
x \\
10 \\
x \\
+ \\
\end{array}$ & $\begin{array}{l}8 \\
m \\
x \\
10 \\
x \\
+\end{array}$ & $\begin{array}{l}0 \\
m \\
x \\
10 \\
\times \\
+\end{array}$ & $\begin{array}{l}\text { v } \\
\dot{x} \\
x \\
10 \\
\times \\
+\end{array}$ & $\begin{array}{l}0 \\
x \\
\dot{x} \\
x \\
\dot{v}\end{array}$ & $\begin{array}{l}\mathbf{N} \\
x \\
b \\
x \\
\dot{v}\end{array}$ & $\begin{array}{l}\infty \\
\vec{x} \\
6 \\
x \\
\dot{v}\end{array}$ & $\begin{array}{l}\vec{y} \\
x \\
x \\
0 \\
x \\
+\end{array}$ & $\begin{array}{l}\dot{m} \\
x \\
\dot{0} \\
x \\
+\end{array}$ & & \\
\hline & & & - & - & - & - & - & . & & 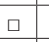 & 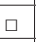 & ㅁ & $\square$ & $\square$ & $\bullet$ & ० & $\begin{array}{ll} \\
\end{array}$ & 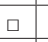 & $\square$ & 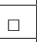 & $\square$ & 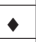 & 0 & $\square$ & \begin{tabular}{l|l}
$\square$ \\
\end{tabular} & 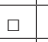 & $\square$ & $\square$ & $\bullet$ & • & $\square$ & $\square$ & $\square$ & $\square$ & \\
\hline & & & - & - & - & - & - & , & & 口 & 口 & 口 & 口 & $\square$ & $\bullet$ & $\circ$ & $\square$ & $\square$ & 口 & $\square$ & 口 & $\bullet$ & & 口 & 口 & $\square$ & 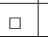 & 口 & 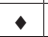 & $\bullet$ & 口 & $\square$ & $\square$ & 口 & \\
\hline PG- & & & - & - & - & - & - & 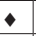 & c & $\square$ & 口 & 口 & 口 & 므 & $\bullet$ & $\circ$ & $\square$ & $\square$ & 다 & & 口 & $\bullet$ & 0 & & 口 & $\square$ & 口 & $\square$ & 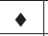 & 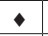 & 口 & ㅁ & 口 & 口 & \\
\hline & & & - & - & - & - & - & 4 & c & - & - & - & - & - & 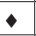 & $\circ$ & ㅁ & - & - & - & - & $\bullet$ & 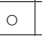 & प & $\square$ & - & - & - & $\bullet$ & 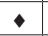 & $\square$ & & - & - & \\
\hline & & - & - & - & - & - & - & & c & $\square$ & 므 & 口 & $\square$ & $\square$ & $\bullet$ & 0 & $\square$ & 다 & $\square$ & 마 & 므 & • & $\circ$ & 口 & $\square$ & $\square$ & $\square$ & $\square$ & $\bullet$ & $\bullet$ & 口 & 口 & 口 & ㅁ & \\
\hline & & & - & & - & - & - & & & - & 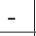 & - & - & - & & & & & & & - & $\bullet$ & & & - & - & - & - & 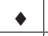 & $\bullet$ & $\square$ & - & - & - & \\
\hline & & & & & - & - & - & & & 도 & $\square$ & & 口 & 마 & 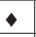 & & ᄃ & & & & ப & 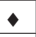 & & & 口 & & & & 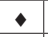 & $\bullet$ & $\square$ & $\square$ & $\square$ & & \\
\hline & & & - & & - & - & - & & c & L & - & - & - & - & & & & & & & - & 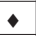 & & & & - & - & & - & 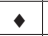 & $\square$ & & & - & \\
\hline G-B & & & & & - & - & - & & $\circ$ & & - & - & - & - & 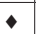 & 0 & 口 & $\square$ & - & - & - & $\bullet$ & 0 & & $\square$ & & & & $\forall$ & 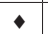 & $\square$ & & & & \\
\hline
\end{tabular}

\begin{tabular}{|c|c|c|c|c|c|c|c|c|c|c|c|c|c|c|c|c|c|c|c|c|c|c|c|c|c|c|c|c|c|c|c|c|c|c|c|}
\hline & & & $\begin{array}{c}x \\
10\end{array}$ & & $\begin{array}{l}x \\
\text { in }\end{array}$ & $\begin{array}{c}x \\
10\end{array}$ & 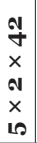 & $\begin{array}{l}0 \\
x \\
m \\
\times \\
10\end{array}$ & $\begin{array}{l}\mathbb{N} \\
x \\
0 \\
x \\
i\end{array}$ & $\begin{array}{l}m \\
x \\
10\end{array}$ & 4 & & $\begin{array}{l}0 \\
m \\
x \\
m \\
x \\
i n\end{array}$ & $\begin{array}{l}\text { I } \\
\times \\
\times \\
m \\
x \\
\text { in }\end{array}$ & $\begin{array}{c}0 \\
x \\
+ \\
x \\
10\end{array}$ & $\begin{array}{c}\mathbf{N} \\
\times \\
+ \\
\times \\
10\end{array}$ & $\begin{array}{l}\infty \\
\vec{x} \\
\vec{\nabla} \\
\times \\
10\end{array}$ & $\begin{array}{l}x \\
10\end{array}$ & $\begin{array}{l}x \\
0 \\
0\end{array}$ & $\begin{array}{l}0 \\
x \\
\dot{+} \\
x \\
10\end{array}$ & 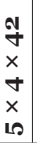 & $\begin{array}{c}0 \\
\times \\
10 \\
\times \\
10\end{array}$ & $\begin{array}{c}\text { N } \\
\times \\
10 \\
\times \\
10\end{array}$ & $\begin{array}{l}\infty \\
\vec{x} \\
10 \\
\times \\
10\end{array}$ & $\begin{array}{l}\mathbf{A} \\
\times \\
10 \\
x \\
10 \\
\end{array}$ & $\begin{array}{l}0 \\
\dot{m} \\
x \\
10 \\
\times \\
10\end{array}$ & $\begin{array}{l}m \\
x \\
10 \\
x \\
10\end{array}$ & $\begin{array}{c}\text { Iี } \\
\times \\
10 \\
x \\
10\end{array}$ & $\begin{array}{l}0 \\
x \\
0 \\
x \\
10\end{array}$ & $\begin{array}{l}\mathbf{J} \\
\times \\
\mathbf{b} \\
\times \\
10\end{array}$ & $\begin{array}{c}\infty \\
\overrightarrow{\vec{x}} \\
0 \\
\times \\
10\end{array}$ & $\begin{array}{l}\mathbb{3} \\
\times \\
0 \\
\times \\
10\end{array}$ & 10 & & \\
\hline & & & & & & - & - & • & 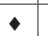 & & 口 & त & 口 & ㅁ & $\bullet$ & 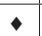 & 0 & & & & 口 & $\bullet$ & $\bullet$ & ০ & 口 & $\square$ & 口 & $\square$ & $\bullet$ & 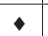 & $\circ$ & $\square$ & $\square$ & 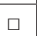 & \\
\hline & & & & & & - & - & $\bullet$ & 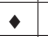 & & & & - & - & $\bullet$ & 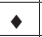 & c & & & & - & $\bullet$ & $\bullet$ & & & & - & - & $\bullet$ & 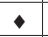 & & & & & \\
\hline & & & & & & - & - & $\bullet$ & • & 도 & ¿ & 口 & ㅁ & $\square$ & $\bullet$ & $\bullet$ & & & & & ㅁ & $\bullet$ & $\bullet$ & 0 & ㅁ & $\square$ & ㅁ & 口 & $\bullet$ & $\bullet$ & & 口 & 口 & 口 & \\
\hline & 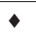 & & - & - & - & - & - & 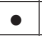 & 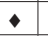 & ㅁ. & ㅁ & ㅁ & $\square$ & $\square$ & $\bullet$ & $\bullet$ & 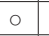 & $\square$ & $L$ & L & ㅁ & $\bullet$ & $\bullet$ & $\circ$ & $\square$ & $\square$ & 口 & 口 & $\bullet$ & 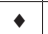 & 0 & 口 & प & 口 & 口 \\
\hline & & & - & - & - & - & - & - & $\cdot$ & 도 & - & - & - & - & $\bullet$ & 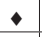 & 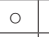 & & & & - & $\bullet$ & 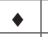 & o & 口 & 口 & - & - & $\bullet$ & 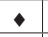 & $\circ$ & $\square$ & 口 & - & \\
\hline & & & & & & - & - & $\bullet$ & . & & ㅁ & & 口 & ㅁ & $\bullet$ & $\bullet$ & & & & & 口 & $\bullet$ & • & & & & ᄂ & & $\bullet$ & $\bullet$ & & & 4 & & \\
\hline & & & & & & - & - & - & 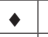 & - & 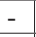 & & - & - & $\bullet$ & $\bullet$ & 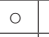 & & & & - & $\bullet$ & $\bullet$ & & & & & & $\bullet$ & $\bullet$ & & & & & \\
\hline & & & - & - & - & - & - & • & . & $\square$ & 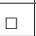 & $\square$ & 口 & 口 & $\bullet$ & $\bullet$ & c & ㅁ & 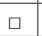 & & 口 & $\bullet$ & 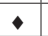 & 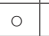 & ㅁ & ㅁ & ㅁ & 口 & $\bullet$ & $\bullet$ & 0 & & 口 & 口 & \\
\hline G-B & & - & - & - & - & - & - & 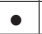 & • & $\square$ & - & - & - & - & $\bullet$ & $\cdot$ & 0 & & - & - & - & $\bullet$ & 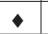 & $\circ$ & $\square$ & $\square$ & $\square$ & & $\bullet$ & 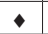 & & & $\square$ & $\square$ & \\
\hline
\end{tabular}

\begin{tabular}{|c|c|c|c|c|c|c|c|c|c|c|c|c|c|c|c|c|c|c|c|c|c|c|c|c|c|c|c|c|c|c|c|c|c|c|}
\hline & & & $\begin{array}{c}\infty \\
\mathbf{x} \\
x \\
\boldsymbol{N} \\
x \\
0 \\
\end{array}$ & $\begin{array}{l}\vec{N} \\
x \\
\mathbf{N} \\
x \\
0 \\
0\end{array}$ & $\begin{array}{l}0 \\
m \\
x \\
\hat{N} \\
x \\
0\end{array}$ & $\begin{array}{l}0 \\
x \\
x \\
N \\
x \\
6\end{array}$ & $\begin{array}{l}\dot{\vec{\gamma}} \\
\times \\
\boldsymbol{N} \\
x \\
\hat{\sigma}\end{array}$ & 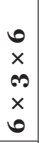 & & $\infty$ & J & $\begin{array}{l}m \\
x \\
m\end{array}$ & $m$ & $\mathscr{F}$ & & & & & $\begin{array}{l}\dot{0} \\
\dot{m} \\
x \\
\dot{+} \\
x \\
0\end{array}$ & $\begin{array}{l}0 \\
m \\
x \\
\dot{\theta} \\
x \\
0\end{array}$ & $\begin{array}{l}\text { चै } \\
\dot{x} \\
x \\
\dot{+} \\
x \\
0 \\
0\end{array}$ & $\begin{array}{l}0 \\
x \\
10 \\
x \\
0 \\
\end{array}$ & \begin{tabular}{c|c} 
N & 0 \\
$x$ & 7 \\
10 & \\
$x$ & \\
0 & 6 \\
\end{tabular} & \begin{tabular}{l|l}
$x$ \\
0 \\
0 & 0
\end{tabular} & $\begin{array}{l}0 \\
\dot{m} \\
x \\
10 \\
x \\
0 \\
0\end{array}$ & $\begin{array}{c}0 \\
0 \\
x \\
10 \\
x \\
0 \\
0\end{array}$ & $\begin{array}{c}\text { yे } \\
\times \\
x \\
10 \\
x \\
\qquad \\
0\end{array}$ & $\begin{array}{l}0 \\
x \\
0 \\
x \\
x \\
0\end{array}$ & \begin{tabular}{l|l} 
N & \\
$\times$ & \\
6 & \\
$x$ & \\
0 &
\end{tabular} & $\begin{array}{l}0 \\
x \\
b\end{array}$ & \begin{tabular}{l|l}
\multirow{N}{*}{} & \\
$x$ & \\
0 & \\
$x$ & \\
0 & \\
\end{tabular} & $\begin{array}{l}0 \\
0 \\
x \\
0 \\
x \\
0 \\
0\end{array}$ & $\begin{array}{l}0 \\
0 \\
x \\
b \\
x \\
0\end{array}$ & \\
\hline & & & 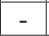 & 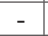 & - & - & & & & & 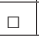 & $\square$ & $\square$ & & & & & c & 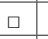 & $\square$ & . & $\bullet$ & $\bullet$ & 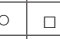 & $\pi$ & $\square$ & $\square$ & $\bullet$ & $\bullet$ & & $\square$ & $\square$ & $\square$ & \\
\hline & & & - & - & 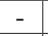 & - & & & & & & & & & & & & & & & & $\bullet$ & & & & & - & & & & & & & \\
\hline & & & - & - & - & - & 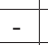 & & & & Б & 口 & 口 & & & & & & 口 & ㅁ & $\square$ & 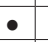 & & & & & $\square$ & $\bullet$ & & & & & & \\
\hline & & & - & - & - & - & 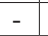 & & & 0 & 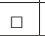 & 口 & ㅁ & & & & & & ㅁ & $\square$ & $\square$ & $\bullet$ & $\bullet$ & & & $\square$ & $\square$ & $\bullet$ & - & & & 口 & & \\
\hline & & • & - & - & - & - & - & 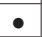 & & 0 & - & - & - & - & & & & & - & - & - & $\bullet$ & $\bullet$ & & & - & - & $\bullet$ & 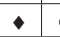 & o & 口 & 口 & 마 & \\
\hline & & - & - & - & 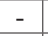 & - & - & - & $\bullet$ & . & $\square$ & 마 & 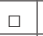 & $\square$ & $\bullet$ & & $e_{0}$ & 다 & 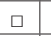 & $\square$ & $\square$ & 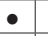 & 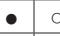 & 0 & $\square$ & $\square$ & $\square$ & $\bullet$ & 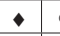 & $\circ$ & $\square$ & $\square$ & $\square$ & \\
\hline & & - & - & - & - & - & 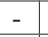 & $\bullet$ & 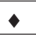 & - & - & - & - & - & • & & 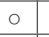 & - & - & - & - & $\bullet$ & $\bullet$ & ㅁ & - & - & - & $\bullet$ & $\bullet$ & $\circ$ & $\square$ & 므 & - & \\
\hline & & & - & - & - & - & - & & & o & 口 & 므 & $\square$ & $\square$ & & & 0 & 므 & 므 & ㅁ & $\square$ & $\bullet$ & $\bullet$ & & 므 & $\square$ & 마 & $\bullet$ & $\bullet$ & 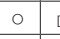 & 므 & ㅁ & 므 & \\
\hline 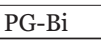 & & & & - & - & - & - & & & & & & & & 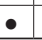 & & & & & 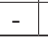 & & $\bullet$ & 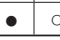 & & & & & $\bullet$ & $\bullet$ & & $\square$ & 므 & $\square$ & \\
\hline
\end{tabular}

$$
\text { - }>30 \% \quad>15 \% \quad \circ>5 \% \quad \square<5 \% \quad-\mathrm{n} / \mathrm{d}
$$


que figuran las normas analizadas y su correspondencia con los diferentes resultados obtenidos (Tabla 5).

En general, aunque las normas analizadas descartan los patios en los que no se pueda inscribir una circunferencia de diámetro inferior a $3 \mathrm{~m}$, se observa que en algunos de estos patios (los de inferior altura) presentan mejores resultados de eficiencia que otros que cumplen esta prescripción.

\section{CONCLUSIONES}

El análisis de la eficiencia de la ventilación de los patios interiores o «de luces» facilita la determinación de la idoneidad del diseño de estos espacios exteriores en razón al uso de los espacios que abren a él.

La gran cantidad de variables que intervienen en el comportamiento del aire libre influyen en mayor o menor medida sobre el desarrollo que tiene el aire en el interior de los patios. La capacidad de intercambio del aire contenido en ellos depende prioritariamente de las condiciones ambientales como la velocidad del viento y de las condiciones constructivas como el entorno, la forma del edificio, la forma y pendiente de la cubierta y el espesor del edificio entre otras. En un esfuerzo de conseguir unos resultados de eficiencia para el diseño general de los patios interiores se ha optado por simplificar las diferentes variables intervinientes a unas condiciones estándar para un edificio aislado con una geometría pura. Se determina así la influencia que el diseño y más concretamente las dimensiones del patio tienen sobre su eficiencia de renovación.

Según los resultados obtenidos, parece lógico plantear patios que presenten alturas de hasta $12 \mathrm{~m}$. O que los espacios residenciales más susceptibles a la calidad del aire (como dormitorios y salas de estar) se abran para fines de ventilación interior únicamente en la mitad superior de patios de altura inferior a los $24 \mathrm{~m}$ y en los primeros $12 \mathrm{~m}$ desde la coronación en el resto de patios. Esto será posible siempre y cuando dos de los cerramientos enfrentados del patio se separen perpendicularmente al menos $5 \mathrm{~m}$, pudiéndose reducir hasta los cuatro metros cuando las aberturas disten verticalmente menos de $6 \mathrm{~m}$ de la coronación del patio.

\section{AGRADECIMIENTOS}

El presente estudio ha sido elaborado gracias al soporte técnico y humano del proyecto de investigación Nacional: El espacio exterior del DB HS3 «Calidad del aire interior»: desarrollo de soluciones alternativas (referencia: VIVIENDA-26562), financiado por el Ministerio de Vivienda y cuya dotación permitió realizar las simulaciones CFD presentadas. Pero en especial agradecemos los datos y series de resultados experimentales puestos a disposición por el Meteorological Institute de Universität Hamburg a través del proyecto CEDVAL para la validación del estudio y en especial al soporte del Dr. Bernd Leitl.

\section{REFERENCIAS}

(1) Skote, M., Sandberg, M., Westerberg, U., Claesson, L., Johansson, A.V. (2005). Numerical and experimental studies of wind environment in an urban morphology. Atmospheric Environment, 39: 6147-6158, doi: http://dx.doi.org/10.1016/j. atmosenv.2005.06.052.

(2) Walker, R.R., Shao, L., Woolliscroft, M. (1993, 21-23 de septiembre). Natural ventilation via courtyards: Theory \& measurements. En 14th AIVC Conference "Energy Impact of Ventilation and Air Infiltration". Copenhague.

(3) Padilla-Marcos, M.A., Feijó-Muñoz, J., Meiss, A. (2015). Wind velocity effects on the quality and efficiency of ventilation in the modelling of outdoor spaces. Case studies. Building Services Engineering Research and Technology, (Published on line first) doi: http://dx.doi.org/10.1177/0143624415596441.

(4) Zaki, S.A., Hagishima, A., Tanimoto, J. (2012). Experimental study of wind-induced ventilation in urban building of cube arrays with various layouts. Journal of Wind Engineering and Industrial Aerodynamics, 103: 31-40, doi: http://dx.doi. org/10.1016/j.jweia.2012.02.008.

(5) Kim, J.J., Baik, J.J. (2004). A numerical study of the effects of ambient wind direction on flow and dispersion in urban street canyons using the RNG k- $\varepsilon$ turbulence model. Atmospheric Environment, 38(19): 3039-3048, doi: http://dx.doi. org/10.1016/j.atmosenv.2004.02.047.

(6) Sandberg, M. (1981). What is ventilation efficiency? Building and Environment, 16(2): 123-135, doi: http://dx.doi. org/10.1016/0360-1323(81)90028-7.

(7) Aldawoud, A. (2013). The influence of the atrium geometry on the building energy performance. Energy and Buildings, 57: 1-5, doi: http://dx.doi.org/10.1016/j.enbuild.2012.10.038.

(8) Buccolieri, R., Sandberg, M., di Sabatino, S. (2011). An application of ventilation efficiency concepts to the analysis of building density effects on urban flow and pollutant concentration. International Journal of Environment and Pollution, 47: 248-256, doi: http://dx.doi.org/10.1504/IJEP.2011.047339.

(9) Shao, L., Walker, R.R., Woolliscroft, M. (1993, 21-23 de septiembre). Natural ventilation via courtyards: The application of CFD. En 14th AIVC Conference "Energy Impact of Ventilation and Air Infiltration". Copenhague.

(10) Padilla-Marcos, M.A., Feijó-Muñoz, J., Meiss, A. (2016). Confined-air quality based on the geometric efficiency of urban outdoor spaces. Cases study. (Artículo de tesis doctoral en prensa). International Journal of Ventilation, 15.

(11) Ministerio de Vivienda. (2006). Real Decreto 314/2006 de 17 de marzo, por el que se aprueba el Código Técnico de la Edificación. Boletín Oficial del Estado, $\mathrm{n}^{0} 74$. España.

(12) Ministerio de la Vivienda. (1969). Orden de 20 de mayo por la que se aprueban las Ordenanzas Provisionales de Viviendas de Protección Oficial. Boletín Oficial del Estado, $\mathrm{n}^{0}{ }^{123}$. España.

(13) Ayuntamiento de Madrid. (1997). Normas Urbanísticas del PGOUM. Boletín Oficial de la Comunidad de Madrid, $\mathrm{n}^{\circ} 92$. España.

(14) Departamento de Territorio y sostenibilidad. Gobierno de Cataluña. (2012). Decreto 141/2012, de 30 de octubre, por el que se regulan las condiciones mínimas de habitabilidad de las viviendas y la cédula de habitabilidad. Diario Oficial de la Generalitat de Cataluña, nº6245. España. 
(15) Ayuntamiento de Barcelona. (1976). Normas Urbanísticas del Plan General Metropolitano de Barcelona. Boletín Oficial de la Provincia de Barcelona del 19/07/1976. España.

(16) Consejería de Vivienda y Ordenación del Territorio. Junta de Andalucía. (2008). Orden de 21 de julio de 2008 , sobre normativa técnica de diseño y calidad aplicable a las viviendas protegidas en la Comunidad Autónoma de Andalucía y se agilizan los procedimientos establecidos para otorgar las Calificaciones de Vivienda Protegidas. Boletín Oficial de la junta de Andalucía, $\mathrm{n}^{\mathrm{0}}{ }_{154}$. España.

(17) Ayuntamiento de Sevilla. (2006). Normas Urbanísticas del Plan General de Ordenación Urbana de Sevilla. Boletín Oficial de la Junta de Andalucía, $\mathrm{n}^{0}{ }_{21}$. España.

(18) Consejería de Vivienda y Asuntos Sociales. Gobierno Vasco. (2009). Orden de 12 de febrero de 2009, por la que se aprueban las Ordenanzas de Diseño de Viviendas de Protección Oficial. Boletín Oficial del País Vasco, nº 43. España

(19) Ayuntamiento de Bilbao. (1995). Plan General de Ordenación Urbana de Bilbao. Boletín Oficial de Bilbao, n¹24. España.

(20) Ayuntamiento de Valladolid. (2004). Plan General de Ordenación Urbana de Valladolid. Boletín Oficial de la Provincia, $n^{0} 48$. España.

(21) Hang, J., Sandberg, M., Li, Y. (2009). Age of air and air exchange efficiency in idealized city models. Building and Environment, 44(8): 1714-1723, doi: http://dx.doi.org/10.1016/j.buildenv.2008.11.013.

(22) Vardoulakis, S., Fisher, B.E.A., Pericleous, K., Gonzalez-Flesca, N. (2003). Modelling air quality in street canyons: a review. Atmospheric Environment, 37(2): 155-182, doi: http://dx.doi.org/10.1016/S1352-2310(02)00857-9.

(23) Jongen, T. (2004). Extension of the Age-of-Fluid Method to Unsteady and Closed-Flow Systems. American Institute of Chemical Engineers (AIChE), 50(9): 2020-2037, doi: http://dx.doi.org/10.1002/aic.10193.

(24) Hertwig, D., Efthimiou, G.C., Bartzis, J.G., Leitl, B. (2012). CFD-RANS model validation of turbulent flow in a semiidealized urban canopy. Journal of Wind Engineering and Industrial Aerodynamics, 111: 61-72, doi: http://dx.doi. org/10.1016/j.jweia.2012.09.003.

(25) Schatzmann, M., Rafailidis, S., Pavageau, M. (1997). Some remarks on the validation of small-scale dispersion models with field and laboratory data. Journal of Wind Engineering and Industrial Aerodynamics, 67-68: 885-893, doi: http:// dx.doi.org/10.1016/So167-6105(97)o0126-8.

(26) Cook, N.J. (1978). Determination of the model scale factor in wind-tunnel simulations of the adiabatic atmospheric boundary layer. Journal of Industrial Aerodynamics, 2(4): 311-321, doi: http://dx.doi.org/10.1016/0167-6105(78)90016-8.

(27) Richards, P.J., Hoxey, R.P. (1993). Appropriate boundary conditions for computational wind engineering models using the $k-\varepsilon$ turbulence model. Journal of Wind Engineering and Industrial Aerodynamics, 46-47: 145-153, doi: http:// dx.doi.org/10.1016/0167-6105(93)90124-7.

(28) Wang, Z.Y., Plate, E.J., Rau, M., Keiser, R. (1996). Scale effects in wind tunnel modelling. Journal of Wind Engineering and Industrial Aerodynamics, 61(2-3): 113-130, doi: http://dx.doi.org/10.1016/0167-6105(96)ooo49-9.

(29) Murakami, S., Hayashi, Y. (1988). 3-D numerical simulation of airflow around a cubic model by means of the k-e model. Journal of Wind Engineering and Industrial Aerodynamics, 31(2-3): 283-303, doi: http://dx.doi.org/10.1016/o1676105(88)90009-8.

(30) Yakhot, V., Orszag, S.A. (1986). Renormalization Group Analysis of Turbulence. Basic Theory. Journal of Scientific Computing, 1(1): 3-51, doi: http://dx.doi.org/10.1007/BF01061452.

(31) Shih, T.H. Liou, W.W., Shabbir, A., Yang, Z., Zhu, J. (1995). A New k- $\varepsilon$ Eddy Viscosity Model for High Reynolds Number Turbulent Flows-Model Development and Validation. Computers \& Fluids, 24(3): 227-238, doi: http://dx.doi. org/10.1016/0045-7930(94)oo032-T.

(32) Li, J., Ward, I.C. (2007). Developing Computational Fluid Dynamics conditions for urban natural ventilation study. En Proceedings: Building Simulation.

(33) Gastón, M., Pascal, E., Frías, L., Martí, I., Irigoyen, U., Cantero, E., Lozano, S., Loureiro, Y. Wind resources map of Spain at mesoscale. Methodology and validation. Sarriguren, Navarra: Centro Nacional de Energías Renovables (CENER).

(34) Sutton, O.G. (1936). The Logarithmic Law of wind Structure near the Ground. Quarterly journal of the Royal Meteorological Society, 63(268): 105-107, doi: http://dx.doi.org/10.1002/qj.49706326820.

(35) Launder, B.E., Spalding, D.B. (1974). The numerical computation of turbulent flows. Computer Methods in Applied Mechanics and Engineering, 3(2): 269-289, doi: http://dx.doi.org/10.1016/0045-7825(74)90029-2.

(36) Hang, J., Sandberg, M., Li, Y. (2009). Effect of urban morphology on wind condition in idealized city models. Atmospheric Environment, 43(4): 869-878, doi: http://dx.doi.org/10.1016/j.atmosenv.2008.10.040. 